\title{
From Dynamical to Numerical R-Matrices: A Case Study for the Calogero Models
}

\author{
Michael Forger and Axel Winterhalder * \\ Departamento de Matemática Aplicada, \\ Instituto de Matemática e Estatística, \\ Universidade de São Paulo, \\ Caixa Postal 66281, \\ BR-05311-970 São Paulo, S.P., Brazil
}

\begin{abstract}
Within the class of integrable Calogero models associated with (semi-) simple Lie algebras and with symmetric pairs of Lie algebras identified in a previous paper, we analyze whether and to what extent it is possible to find a gauge transformation that takes the traditional Lax pair with its dynamical $R$-matrix to a new Lax pair with a numerical $R$-matrix.
\end{abstract}

Universidade de São Paulo

RT-MAP-0202

December 2002

\footnotetext{
*forger@ime.usp.br and winter@ime.usp.br

*Work supported by CNPq (Conselho Nacional de Desenvolvimento Científico e Tecnológico), Brazil, by FAPESP (Fundação de Amparo à Pesquisa do Estado de São Paulo), Brazil.
} 


\section{Introduction}

In a recent paper [1], we have performed a systematic analysis of the Calogero-MoserSutherland models, or Calogero models, for short, which constitute an important class of completely integrable Hamiltonian systems. Our work follows the traditional Lie algebraic approach outlined long ago by Olshanetsky and Perelomov [2, 3, 4] which is based on the use of (semi-) simple Lie algebras and, more generally, of symmetric pairs, extending it so as to encompass the construction not only of a Lax representation for the equations of motion but also that of a dynamical $R$-matrix. The existence of these structures was found to depend on the possibility of solving a simple set of algebraic constraints for a certain function $F$ or $K$ that assigns to each root $\alpha$ a generator $F_{\alpha}$ or $K_{\alpha}$ in the pertinent Cartan subalgebra $\mathfrak{h}:$ these state that for any two roots $\alpha$ and $\beta$

$$
g_{\alpha} \alpha\left(F_{\beta}\right)-g_{\beta} \beta\left(F_{\alpha}\right)=\Gamma_{\alpha, \beta}
$$

in the case of (semi-) simple Lie algebras $\mathfrak{g}$ and

$$
g_{\alpha} \alpha\left(K_{\beta}\right)-g_{\beta} \beta\left(K_{\alpha}\right)=\Gamma_{\alpha, \beta}^{\theta}
$$

in the case of symmetric pairs $(\mathfrak{g}, \theta)$, where the coefficients $\Gamma_{\alpha, \beta}$ and $\Gamma_{\alpha, \beta}^{\theta}$ are defined in terms of the structure constants $N_{\alpha, \beta}$ of $\mathfrak{g}$ and the coupling constants $g_{\alpha}$ of the model by

$$
\Gamma_{\alpha, \beta}=g_{\alpha+\beta} N_{\alpha, \beta}
$$

and by

$$
\Gamma_{\alpha, \beta}^{\theta}=\frac{1}{4}\left(g_{\alpha+\beta} N_{\alpha, \beta}+g_{\theta \alpha+\beta} N_{\theta \alpha, \beta}+g_{\alpha+\theta \beta} N_{\alpha, \theta \beta}+g_{\theta \alpha+\theta \beta} N_{\theta \alpha, \theta \beta}\right)
$$

respectively.2] In the first case, it was found that a solution of these constraints exists only for the Lie algebras $\mathfrak{s l}(n, \mathbb{C})$ of the $A$-series, whereas in the second case, explicit solutions were found for the complex Grassmannians $S U(p+q) / S(U(p) \times U(q))$ of the $A I I I$ - series when $|p-q| \leqslant 1$.

Following a somewhat different direction, several authors [5, 6] have recently shown by explicit matrix computations that the Calogero models based on $\mathfrak{s l}(n, \mathbb{C})$, degenerate as well as elliptic, admit a gauge transformation taking the dynamical $R$-matrix into a numerical one: this is achieved by explicitly constructing a group-valued function on the configuration space which is used to conjugate the standard Lax pair and dynamical $R$-matrix of the model into a new Lax pair and a numerical $R$-matrix.

\footnotetext{
${ }^{1}$ In this paper, we adopt a slightly modified notation: for reasons to become clear towards the end of the paper, we shall in the case of symmetric pairs denote the generators $F_{\alpha}$ of Ref. [1] by $K_{\alpha}$.

${ }^{2}$ In the case of symmetric pairs, it is also assumed that the root generators $E_{\alpha}$ in $\mathfrak{g}$ can be and have been chosen so that $\theta E_{\alpha}=E_{\theta \alpha}$ for all $\alpha \in \Delta$, implying that $N_{\theta \alpha, \theta \beta}=N_{\alpha, \beta}$ for all $\alpha, \beta \in \Delta$. As explained in the erratum to Ref. [1], this is not always possible but is a necessary condition for our proof of integrability, and it can always be arranged to hold for all roots $\alpha$ if it can be made to hold for all real roots $\alpha$, that is, all roots $\alpha$ in $\Delta$ satisfying $\theta \alpha=-\alpha$.
} 
In the present paper, we systematize the method of Fehér and Pusztai [6], adapting it to the formalism developed in our previous work [1]: this allows us to extend it from the degenerate to the elliptic models as well as from the case of Lie algebras to that of symmetric pairs. In all cases, we find that the existence of a gauge transformation with the desired property can be reduced to a set of purely algebraic constraints which are similar to but not identical with the integrability constraints (1) and (2) found in Ref. [1]. In the case of Lie algebras, it turns out that these various constraints all have one and the same solution, thus confirming the previous results of other authors [5, 6] that the well-known dynamical $R$-matrices of the Calogero models based on $\mathfrak{s l}(n, \mathbb{C})$ can be gauge transformed to numerical $R$-matrices. In the case of symmetric pairs, however, we find extra constraints on the root system, over and above those that guarantee integrability. In particular, for the $A I I I$-series of complex complex Grassmannians $S U(p+q) / S(U(p) \times U(q))$ where integrability has in Ref. [1] been shown to hold when $|p-q| \leqslant 1$, these constraints exclude the case $|p-q|=1$ but allow for a solution in the case $p=q$. This means that the dynamical $R$-matrices for the Calogero models associated with the classical root systems can be gauge transformed to numerical $R$ matrices for the $C_{n}$ and $D_{n}$ systems but not for the $B_{n}$ and $B C_{n}$ systems; remarkably, the latter are just the ones containing an explicit dependence on the coupling constants.

The paper is organized as follows. In Sect. 2, we give a brief summary of the method of gauge transforming Lax pairs and dynamical $R$-matrices in integrable systems such as the Calogero models; moreover, we collect a number of identities to be used repeatedly later on. In Sect. 3, we present our calculations for the case of Lie algebras and in Sect. 4 those for symmetric pairs. Finally, in Sect. 5 we draw our conclusions and comment on perspectives for further work.

\section{Gauge Transformations}

Consider an integrable model with a finite-dimensional phase space which we assume to be the cotangent bundle $T^{*} Q$ of a configuration space $Q$. Integrability is encoded into the existence of a Lax representation for the equations of motion,

$$
\dot{L}=[L, M]
$$

together with that of an $R$-matrix whose role is to control the Poisson brackets between the components of the Lax matrix $L$, according to the formula [7]

$$
\left\{L_{1}, L_{2}\right\}=\left[R_{12}, L_{1}\right]-\left[R_{21}, L_{2}\right]
$$

Here, $L$ and $M$ are maps from $T^{*} Q$ into a given Lie algebra $\mathfrak{g}$ whereas $R$ will in general be a map from $T^{*} Q$ into the second tensor power $U(\mathfrak{g}) \otimes U(\mathfrak{g})$ of the universal enveloping algebra $U(\mathfrak{g})$ of $\mathfrak{g}$; as usual, $L_{1}=L \otimes 1, L_{2}=1 \otimes L$ etc.. The choice of

$\mathfrak{g}$ is far from obvious; it reflects the hidden symmetries that are present in the model. 
Moreover, even if one fixes $\mathfrak{g}$ and a connected Lie group $G$ that has $\mathfrak{g}$ as its Lie algebra, $L, M$ and $R$ are not uniquely determined. In particular, we are free to perform a gauge transformation by an arbitrary function $g$ on $T^{*} Q$ with values in $G$, as follows:

$$
\begin{gathered}
L^{\prime}=g L g^{-1}, \\
R_{12}^{\prime}=g_{1} g_{2}\left(R_{12}+g_{1}^{-1}\left\{g_{1}, L_{2}\right\}+\frac{1}{2}\left[g_{1}^{-1} g_{2}^{-1}\left\{g_{1}, g_{2}\right\}, L_{2}\right]\right) g_{1}^{-1} g_{2}^{-1} .
\end{gathered}
$$

The second transformation law is dictated by the requirement that the fundamental Poisson bracket relation (6) should be preserved under this transformation, which is easy to check. Note that in general, $L, M$ and $g$ may depend on a spectral parameter $u$, in which case $R$ will depend on two spectral parameters $u$ and $v$.

In the case of the Calogero models of interest here, $\mathfrak{g}$ is a simple complex Lie algebra, with Cartan subalgebra $\mathfrak{h}$ and corresponding root system $\Delta$ fixed once and for all, $Q$ is an open subset in a real subspace of $\mathfrak{h}$ in which we fix a basis $\left\{H_{1}, \ldots, H_{r}\right\}, L$ is of the form

$$
L(q, p ; u)=\sum_{j=1}^{r} p_{j} H_{j}+\sum_{\alpha \in \Delta} L_{\alpha}(q, u) E_{\alpha},
$$

with functions $L_{\alpha}$ whose explicit form will be needed only later, and $R$ is independent of the momentum variables. (For details, see Ref. [G].) Our aim in what follows will be to determine $g$ in such a way that $R^{\prime}$ becomes constant (as a function on phase space). To this end, we shall assume that $g$ is also independent of the momentum variables and introduce the "gauge potentials"

$$
A_{j}(u)=g^{-1}(u) \partial_{j} g(u) .
$$

Reverting to ordinary tensor notation, we get

$$
\left(g^{-1}(u) \otimes 1\right)\{g(u) \otimes, L(v)\}=-\sum_{j=1}^{r} g^{-1}(u)\left\{p_{j}, g(u)\right\} \otimes H_{j}=-\sum_{j=1}^{r} A_{j}(u) \otimes H_{j},
$$

so eqn (8) simplifies to

$$
R^{\prime}(u, v)=(g(u) \otimes g(v))\left(R(u, v)-\sum_{j=1}^{r} A_{j}(u) \otimes H_{j}\right)\left(g^{-1}(u) \otimes g^{-1}(v)\right) .
$$

This implies

$$
\begin{aligned}
\left(g^{-1}(u) \otimes\right. & \left.g^{-1}(v)\right) \partial_{k} R^{\prime}(u, v)(g(u) \otimes g(v)) \\
= & \partial_{k}\left(R(u, v)-\sum_{j=1}^{r} A_{j}(u) \otimes H_{j}\right) \\
& +\left[\left(g^{-1}(u) \otimes g^{-1}(v)\right) \partial_{k}(g(u) \otimes g(v)), R(u, v)-\sum_{j=1}^{r} A_{j}(u) \otimes H_{j}\right],
\end{aligned}
$$


so the condition that the partial derivatives $\partial_{k} R^{\prime}(u, v)$ of $R^{\prime}(u, v)$ all vanish amounts to requiring

$$
\begin{aligned}
\partial_{k}(R(u, v) & \left.-\sum_{j=1}^{r} A_{j}(u) \otimes H_{j}\right) \\
- & {\left[R(u, v)-\sum_{j=1}^{r} A_{j}(u) \otimes H_{j}, A_{k}(u) \otimes 1+1 \otimes A_{k}(v)\right]=0 . }
\end{aligned}
$$

Using the integrability condition

$$
\partial_{k} A_{l}(u)-\partial_{l} A_{k}(u)+\left[A_{k}(u), A_{l}(u)\right]=0
$$

that follows from eqn (10), this can be rewritten in the form

$$
\begin{aligned}
& \partial_{k} R(u, v)-\sum_{j=1}^{r} \partial_{j} A_{k}(u) \otimes H_{j} \\
& -\left[R(u, v), A_{k}(u) \otimes 1+1 \otimes A_{k}(v)\right]+\sum_{j=1}^{r} A_{j}(u) \otimes\left[H_{j}, A_{k}(v)\right]=0 .
\end{aligned}
$$

In order to compute the content of eqns (11) and (12), we shall in what follows expand the gauge potential according to

$$
A_{j}(u)=A_{j}^{\mathfrak{h}}(u)+\sum_{\alpha \in \Delta} A_{j}^{\alpha}(u) E_{\alpha}
$$

which allows us, in particular, to decompose eqn (11) into its Cartan part

$$
\partial_{k} A_{l}^{\mathfrak{h}}(u)-\partial_{l} A_{k}^{\mathfrak{h}}(u)+\sum_{\alpha \in \Delta} A_{k}^{\alpha}(u) A_{l}^{-\alpha}(u) H_{\alpha}=0
$$

and its root part

$$
\begin{aligned}
\partial_{k} A_{l}^{\alpha}(u)-\partial_{l} A_{k}^{\alpha}(u) & +\alpha\left(A_{k}^{\mathfrak{h}}(u)\right) A_{l}^{\alpha}(u)-\alpha\left(A_{l}^{\mathfrak{h}}(u)\right) A_{k}^{\alpha}(u) \\
& +\sum_{\substack{\beta, \gamma \in \Delta \\
\beta+\gamma=\alpha}} N_{\beta, \gamma} A_{k}^{\beta}(u) A_{l}^{\gamma}(u)=0 .
\end{aligned}
$$

In what follows, we shall analyze under what conditions this system of equations admits solutions when we insert the explicit expressions for $R$ given in Ref. [1] and evaluate the commutators in eqn (12) using the usual abbreviation $\alpha_{j}=\alpha\left(H_{j}\right)$ and the relations

$$
\begin{aligned}
& {\left[H_{j} \otimes H_{j}, E_{\alpha} \otimes 1\right]=\alpha_{j} E_{\alpha} \otimes H_{j},} \\
& {\left[H_{j} \otimes H_{j}, 1 \otimes E_{\alpha}\right]=\alpha_{j} H_{j} \otimes E_{\alpha},}
\end{aligned}
$$


(no summation over $j$ ),

$$
\begin{gathered}
{\left[F_{\gamma} \otimes E_{\gamma}, H_{j} \otimes 1\right]=0} \\
{\left[F_{\gamma} \otimes E_{\gamma}, 1 \otimes H_{j}\right]=-\gamma_{j} F_{\gamma} \otimes E_{\gamma},} \\
{\left[F_{\gamma} \otimes E_{\gamma}, E_{\delta} \otimes 1\right]=\delta\left(F_{\gamma}\right) E_{\delta} \otimes E_{\gamma},} \\
{\left[F_{\gamma} \otimes E_{\gamma}, 1 \otimes E_{\gamma}\right]=0,} \\
{\left[F_{\gamma} \otimes E_{\gamma}, 1 \otimes E_{-\gamma}\right]=F_{\gamma} \otimes H_{\gamma},} \\
{\left[F_{\gamma} \otimes E_{\gamma}, 1 \otimes E_{\delta}\right]=N_{\gamma, \delta} F_{\gamma} \otimes E_{\gamma+\delta} \text { if } \gamma \pm \delta \neq 0}
\end{gathered}
$$

(valid for any set of generators $F_{\gamma}$ belonging to the Cartan subalgebra $\mathfrak{h}$ ) and

$$
\begin{gathered}
{\left[E_{\gamma} \otimes E_{-\gamma}, H_{j} \otimes 1\right]=-\gamma_{j} E_{\gamma} \otimes E_{-\gamma},} \\
{\left[E_{\gamma} \otimes E_{-\gamma}, 1 \otimes H_{j}\right]=\gamma_{j} E_{\gamma} \otimes E_{-\gamma},} \\
{\left[E_{\gamma} \otimes E_{-\gamma}, E_{\gamma} \otimes 1\right]=0} \\
{\left[E_{\gamma} \otimes E_{-\gamma}, E_{-\gamma} \otimes 1\right]=H_{\gamma} \otimes E_{-\gamma},} \\
{\left[E_{\gamma} \otimes E_{-\gamma}, E_{\delta} \otimes 1\right]=N_{\gamma, \delta} E_{\gamma+\delta} \otimes E_{-\gamma} \quad \text { if } \gamma \pm \delta \neq 0} \\
{\left[E_{\gamma} \otimes E_{-\gamma}, 1 \otimes E_{\gamma}\right]=-E_{\gamma} \otimes H_{\gamma},} \\
{\left[E_{\gamma} \otimes E_{-\gamma}, 1 \otimes E_{-\gamma}\right]=0,} \\
{\left[E_{\gamma} \otimes E_{-\gamma}, 1 \otimes E_{\delta}\right]=N_{-\gamma, \delta} E_{\gamma} \otimes E_{-\gamma+\delta} \quad \text { if } \gamma \pm \delta \neq 0,}
\end{gathered}
$$

The second step would be to determine $g$ itself and, from there, find $L^{\prime}$ and $R^{\prime}$ : this question will be addressed elsewhere in order not to overload our presentation here.

Concluding this section, let us for later use collect the functional identities satisfied by the coefficient functions $L_{\alpha}$ that appear in eqn (9) above. For the degenerate models, $L_{\alpha}(q, u)=\mathrm{i} g_{\alpha} w(\alpha(q))$ where $w$ is an odd function of its argument,

$$
w(-t)=-w(t)
$$

that satisfies the differential equation

$$
\left(\frac{w^{\prime}}{w}\right)^{\prime}=w^{2}
$$

as well as the functional equation

$$
\left(\frac{w^{\prime}(s)}{w(s)}+\frac{w^{\prime}(t)}{w(t)}\right) w(s+t)+w(s) w(t)=0
$$


already employed in Ref. [1]. For the elliptic models, $L_{\alpha}(q, u)=\mathrm{i} g_{\alpha} \Phi(\alpha(q), u)$ where $\Phi$ and the closely related Weierstrass zeta function satisfy the symmetry properties

$$
\Phi\left(-z_{1},-z_{2}\right)=-\Phi\left(z_{1}, z_{2}\right) \quad, \quad \zeta(-z)=-\zeta(z)
$$

and the functional equations

$$
\begin{gathered}
\Phi(s, u) \Phi(-s, u)=\zeta^{\prime}(s)-\zeta^{\prime}(u), \\
\Phi(s, u) \Phi^{\prime}(-s, u)-\Phi^{\prime}(s, u) \Phi(-s, u)=-\zeta^{\prime \prime}(s), \\
\Phi(s, u) \Phi^{\prime}(t, u)-\Phi^{\prime}(s, u) \Phi(t, u)=-\left(\zeta^{\prime}(s)-\zeta^{\prime}(t)\right) \Phi(s+t, u), \\
\Phi(-s, v-u) \Phi(s+t, v)+\Phi(-t, u-v) \Phi(s+t, u)=-\Phi(s, u) \Phi(t, v), \\
\Phi(-s, u-v) \Phi(s, u)+(\zeta(v-u)+\zeta(u)) \Phi(s, v)=\Phi^{\prime}(s, v),
\end{gathered}
$$

already employed in Ref. [1], as well as the additional functional equations

$$
\begin{gathered}
\Phi^{\prime}(s, u)=(\zeta(s+u)-\zeta(s)) \Phi(s, u) \\
\Phi(s, u) \Phi(t, u)=(\zeta(s)+\zeta(t)+\zeta(u)-\zeta(s+t+u)) \Phi(s+t, u),
\end{gathered}
$$

where $\Phi^{\prime}$ denotes the derivative of $\Phi$ with respect to the first argument; all of these can be derived from the representation of $\Phi$ and $\zeta$ in terms of the Weierstrass $\sigma$ function:

$$
\Phi\left(z_{1}, z_{2}\right)=\frac{\sigma\left(z_{1}+z_{2}\right)}{\sigma\left(z_{1}\right) \sigma\left(z_{2}\right)} \quad, \quad \zeta(z)=\frac{\sigma^{\prime}(z)}{\sigma(z)}
$$

Note that in the degenerate case, the spectral parameter drops out. In fact, all of the calculations to be presented in what follows can be carried out for the degenerate case in exactly the same manner as for the elliptic case, provided one performs the following substitutions:

$$
\begin{array}{ccc}
\Phi(s, u), \Phi(s, v) & \rightarrow & w(s) \\
\Phi(s, u-v), \Phi(s, v-u), \zeta(s) & \rightarrow & -\frac{w^{\prime}(s)}{w(s)} \\
\zeta(u), \zeta(v), \zeta(u-v), \zeta(v-u) & & 0 \\
\zeta(s+u), \zeta(s+t+u) & \rightarrow &
\end{array}
$$

Therefore, we shall suppress the calculations for the degenerate models, except at the few points where substantial differences arise. 


\section{Calogero Models for Semisimple Lie Algebras}

According to Ref. [1], the standard Lax matrix $L$ and the dynamical $R$-matrix for the Calogero models associated with the root system $\Delta$ of a simple complex Lie algebra $\mathfrak{g}$ read

$$
\begin{gathered}
L=\sum_{j=1}^{r} p_{j} H_{j}+\sum_{\alpha \in \Delta} \mathrm{i} g_{\alpha} w(\alpha(q)) E_{\alpha} \\
R=\sum_{\alpha \in \Delta} w(\alpha(q)) F_{\alpha} \otimes E_{\alpha}+\sum_{\alpha \in \Delta} \frac{w^{\prime}(\alpha(q))}{w(\alpha(q))} E_{\alpha} \otimes E_{-\alpha},
\end{gathered}
$$

for the degenerate model and

$$
\begin{aligned}
L(u)= & \sum_{j=1}^{r} p_{j} H_{j}+\sum_{\alpha \in \Delta} \mathrm{i} g_{\alpha} \Phi(\alpha(q), u) E_{\alpha}, \\
R(u, v)= & -\sum_{j=1}^{r}(\zeta(u-v)+\zeta(v)) H_{j} \otimes H_{j} \\
& +\sum_{\alpha \in \Delta} \Phi(\alpha(q), v) F_{\alpha} \otimes E_{\alpha} \\
& -\sum_{\alpha \in \Delta} \Phi(\alpha(q), u-v) E_{\alpha} \otimes E_{-\alpha}
\end{aligned}
$$

for the elliptic model. As has been shown in Ref. [1], integrability requires the generators $F_{\alpha} \in \mathfrak{h}_{\mathbb{R}}$ appearing in eqns (34) and (36) to satisfy the constraints (11). Moreover, writing

$$
F_{\alpha}^{ \pm}=\frac{1}{2}\left(F_{\alpha} \pm F_{-\alpha}\right)
$$

we also impose the condition

$$
\alpha\left(F_{\alpha}^{+}\right)=0
$$

which follows from eqn (1) by setting $\beta=-\alpha$ when $g_{\alpha} \neq 0$ but turns out to be true in general, independent of this hypothesis.

In order to compute the content of eqns (11) and (12), we further expand the Cartan part of the gauge potential according to

$$
A_{j}^{\mathfrak{h}}(u)=\sum_{k=1}^{r} A_{j}^{k}(u) H_{k} .
$$

Then inserting eqns (36), (13) and (39) into eqn (12), we obtain 


$$
\begin{aligned}
& 0=\sum_{\alpha \in \Delta} \alpha_{k} \Phi^{\prime}(\alpha(q), v) F_{\alpha} \otimes E_{\alpha}-\sum_{\alpha \in \Delta} \alpha_{k} \Phi^{\prime}(\alpha(q), u-v) E_{\alpha} \otimes E_{-\alpha} \\
& -\sum_{j=1}^{r} \partial_{j} A_{k}^{\mathfrak{h}}(q, u) \otimes H_{j}-\sum_{j=1}^{r} \sum_{\alpha \in \Delta} \partial_{j} A_{k}^{\alpha}(q, u) E_{\alpha} \otimes H_{j} \\
& +\sum_{j=1}^{r} \sum_{\alpha \in \Delta}(\zeta(u-v)+\zeta(v)) A_{k}^{\alpha}(q, u)\left[H_{j} \otimes H_{j}, E_{\alpha} \otimes 1\right] \\
& +\sum_{j=1}^{r} \sum_{\alpha \in \Delta}(\zeta(u-v)+\zeta(v)) A_{k}^{\alpha}(q, v)\left[H_{j} \otimes H_{j}, 1 \otimes E_{\alpha}\right] \\
& -\sum_{j=1}^{r} \sum_{\gamma \in \Delta} \Phi(\gamma(q), v) A_{k}^{j}(q, u)\left[F_{\gamma} \otimes E_{\gamma}, H_{j} \otimes 1\right] \\
& -\sum_{j=1}^{r} \sum_{\gamma \in \Delta} \Phi(\gamma(q), v) A_{k}^{j}(q, v)\left[F_{\gamma} \otimes E_{\gamma}, 1 \otimes H_{j}\right] \\
& -\sum_{\gamma, \delta \in \Delta} \Phi(\gamma(q), v) A_{k}^{\delta}(q, u)\left[F_{\gamma} \otimes E_{\gamma}, E_{\delta} \otimes 1\right] \\
& -\sum_{\gamma, \delta \in \Delta} \Phi(\gamma(q), v) A_{k}^{\delta}(q, v)\left[F_{\gamma} \otimes E_{\gamma}, 1 \otimes E_{\delta}\right] \\
& +\sum_{j=1}^{r} \sum_{\gamma \in \Delta} \Phi(\gamma(q), u-v) A_{k}^{j}(q, u)\left[E_{\gamma} \otimes E_{-\gamma}, H_{j} \otimes 1\right] \\
& +\sum_{j=1}^{r} \sum_{\gamma \in \Delta} \Phi(\gamma(q), u-v) A_{k}^{j}(q, v)\left[E_{\gamma} \otimes E_{-\gamma}, 1 \otimes H_{j}\right] \\
& +\sum_{\gamma, \delta \in \Delta} \Phi(\gamma(q), u-v) A_{k}^{\delta}(q, u)\left[E_{\gamma} \otimes E_{-\gamma}, E_{\delta} \otimes 1\right] \\
& +\sum_{\gamma, \delta \in \Delta} \Phi(\gamma(q), u-v) A_{k}^{\delta}(q, v)\left[E_{\gamma} \otimes E_{-\gamma}, 1 \otimes E_{\delta}\right] \\
& +\sum_{j=1}^{r} \sum_{\alpha \in \Delta} \alpha_{j} A_{k}^{\alpha}(q, v) A_{j}^{\mathfrak{h}}(q, u) \otimes E_{\alpha}+\sum_{j=1}^{r} \sum_{\alpha, \beta \in \Delta} \beta_{j} A_{j}^{\alpha}(q, u) A_{k}^{\beta}(q, v) E_{\alpha} \otimes E_{\beta} .
\end{aligned}
$$

Using eqns (16)-(20) to carry out the commutators, together with the relation

$$
\sum_{j=1}^{r} \alpha_{j} H_{j}=H_{\alpha}
$$

we can collect the terms to identify the components of eqn (12) along the various subspaces of $\mathfrak{g} \otimes \mathfrak{g}$ : 
those along $\mathfrak{h} \otimes H_{j}(1 \leqslant j \leqslant r)$,

$$
\partial_{j} A_{k}^{\mathfrak{h}}(q, u)+\sum_{\alpha \in \Delta} \alpha_{j} \Phi(\alpha(q), v) A_{k}^{-\alpha}(q, v) F_{\alpha}=0
$$

those along $\mathfrak{h} \otimes \mathfrak{g}_{\alpha}(\alpha \in \Delta)$,

$$
\begin{aligned}
\alpha_{k} \Phi^{\prime}(\alpha(q), v) F_{\alpha} & +(\zeta(u-v)+\zeta(v)) A_{k}^{\alpha}(q, v) H_{\alpha}+\Phi(\alpha(q), v) \alpha\left(A_{k}^{\mathfrak{h}}(q, v)\right) F_{\alpha} \\
& -\sum_{\substack{\gamma, \delta \in \Delta \\
\gamma+\delta=\alpha}} N_{\gamma, \delta} \Phi(\gamma(q), v) A_{k}^{\delta}(q, v) F_{\gamma} \\
& -\Phi(-\alpha(q), u-v) A_{k}^{\alpha}(q, u) H_{\alpha}+\sum_{j=1}^{r} \alpha_{j} A_{k}^{\alpha}(q, v) A_{j}^{\mathfrak{h}}(q, u)=0
\end{aligned}
$$

those along $\mathfrak{g}_{\alpha} \otimes H_{j}(\alpha \in \Delta, 1 \leqslant j \leqslant r)$,

$$
\partial_{j} A_{k}^{\alpha}(q, u)+\alpha_{j} \Phi(\alpha(q), u-v) A_{k}^{\alpha}(q, v)-\alpha_{j}(\zeta(u-v)+\zeta(v)) A_{k}^{\alpha}(q, u)=0,
$$

those along $\mathfrak{g}_{\alpha} \otimes \mathfrak{g}_{\alpha}(\alpha \in \Delta)$,

$$
\alpha\left(F_{\alpha}\right) \Phi(\alpha(q), v) A_{k}^{\alpha}(q, u)-\sum_{j=1}^{r} \alpha_{j} A_{j}^{\alpha}(q, u) A_{k}^{\alpha}(q, v)=0
$$

those along $\mathfrak{g}_{\alpha} \otimes \mathfrak{g}_{-\alpha}(\alpha \in \Delta)$,

$$
\begin{aligned}
\alpha_{k} \Phi^{\prime}(\alpha(q), u-v) & +\alpha\left(F_{-\alpha}\right) \Phi(-\alpha(q), v) A_{k}^{\alpha}(q, u) \\
& +\Phi(\alpha(q), u-v)\left(\alpha\left(A_{k}^{\mathfrak{h}}(q, u)\right)-\alpha\left(A_{k}^{\mathfrak{h}}(q, v)\right)\right) \\
& +\sum_{j=1}^{r} \alpha_{j} A_{j}^{\alpha}(q, u) A_{k}^{-\alpha}(q, v)=0
\end{aligned}
$$

and finally those along $\mathfrak{g}_{\alpha} \otimes \mathfrak{g}_{\beta}$ with $\alpha, \beta \in \Delta, \alpha \pm \beta \neq 0$,

$$
\begin{aligned}
& \alpha\left(F_{\beta}\right) \Phi(\beta(q), v) A_{k}^{\alpha}(q, u) \\
& -N_{\alpha, \beta}\left(\Phi(\alpha(q), u-v) A_{k}^{\alpha+\beta}(q, v)-\Phi(-\beta(q), u-v) A_{k}^{\alpha+\beta}(q, u)\right) \\
& -\sum_{j=1}^{r} \beta_{j} A_{j}^{\alpha}(q, u) A_{k}^{\beta}(q, v)=0 .
\end{aligned}
$$

This is a complicated set of equations which we shall solve in a series of steps.

We begin by considering the differential equation (43) for the root part of the gauge potential, which by using the functional equation (29) (with $u$ and $v$ interchanged) is seen to have the simple solution

$$
A_{k}^{\alpha}(q, u)=\Phi(\alpha(q), u) a_{k}^{\alpha}
$$


where the coefficients $a_{k}^{\alpha}$ are constants that must be determined from the remaining equations. For what follows, we shall find it convenient to assemble these constants into a vector in $\mathfrak{h}_{\mathbb{R}}$ by writing, for any $\alpha \in \Delta$,

$$
a_{\alpha}=\sum_{j=1}^{r} a_{j}^{\alpha} H_{j}
$$

so that of course

$$
a_{k}^{\alpha}=\left(H_{k}, a_{\alpha}\right) .
$$

In analogy with eqn (37), we also introduce the abbreviation

$$
a_{\alpha}^{ \pm}=\frac{1}{2}\left(a_{\alpha} \pm a_{-\alpha}\right)
$$

Now we are ready to state the first main result of this section.

Proposition 1 The integrable Calogero model associated with the root system of a simple complex Lie algebra $\mathfrak{g}$ admits a gauge transformation $g$ from the standard Lax pair of Olshanetsky and Perelomov and the dynamical R-matrix of Ref. [1], as given by eqns (33-30), to a new Lax pair with a numerical $R$-matrix if and only if the set of generators $F_{\alpha} \in \mathfrak{h}_{\mathbb{R}}$ appearing in eqns (34) and (30) satisfies the algebraic constraints

$$
\begin{gathered}
\alpha\left(F_{\alpha}^{+}\right)=0, \\
F_{\alpha}^{-}=\frac{\epsilon_{\alpha}}{\sqrt{2}|\alpha|} H_{\alpha}, \\
\alpha\left(F_{\beta}\right) F_{\alpha}-\beta\left(F_{\alpha}\right) F_{\beta}=N_{\alpha, \beta} F_{\alpha+\beta} \\
\text { for } \alpha, \beta \in \Delta \text { such that } \beta \neq \pm \alpha,
\end{gathered}
$$

as well as the additional algebraic constraints

$$
\begin{gathered}
\sum_{\alpha \in \Delta} H_{\alpha} \otimes F_{\alpha} \otimes F_{-\alpha}=0 \\
\sum_{\substack{\beta, \gamma \in \Delta \\
\beta+\gamma=\alpha}} N_{\beta, \gamma} F_{\beta} \otimes F_{\gamma}=H_{\alpha} \otimes F_{\alpha}-F_{\alpha} \otimes H_{\alpha},
\end{gathered}
$$

to be imposed in the case of the elliptic model, where $F_{\alpha}^{ \pm}=\frac{1}{2}\left(F_{\alpha} \pm F_{-\alpha}\right)$ as above, with $\epsilon_{\alpha}= \pm 1$. In this case, the root part and the Cartan part of the gauge potential $A_{k}=g^{-1} \partial_{k} g$ associated with this gauge transformation $g$ are given by

$$
A_{k}^{\alpha}(q)=w(\alpha(q))\left(H_{k}, F_{\alpha}\right)
$$

and

$$
A_{k}^{\mathfrak{h}}(q)=\sum_{\alpha \in \Delta} \frac{w^{\prime}(\alpha(q))}{w(\alpha(q))}\left(H_{k}, F_{-\alpha}\right) F_{\alpha}
$$


for the degenerate model and by

$$
A_{k}^{\alpha}(q, u)=\Phi(\alpha(q), u)\left(H_{k}, F_{\alpha}\right),
$$

and

$$
A_{k}^{\mathfrak{h}}(q, u)=-\sum_{\alpha \in \Delta} \zeta(\alpha(q))\left(H_{k}, F_{-\alpha}\right) F_{\alpha}-\zeta(u) H_{k},
$$

for the elliptic model.

Note. As we shall show after completing the proof of Proposition 1, eqn (53) forces all roots $\alpha$ in $\Delta$ to have the same length (which by convention we fix to be $\sqrt{2}$ ) and also allows for a choice of basis in which the signs $\epsilon_{\alpha}$ are independent of $\alpha$. so that eqn (52) can be simplified as follows:

$$
F_{\alpha}^{-}=\frac{1}{2} \epsilon H_{\alpha} .
$$

Proof. With the vector notation introduce above, we can first of all reduce eqn (44) to a single algebraic constraint:

$$
\alpha\left(a_{\alpha}\right)=\alpha\left(F_{\alpha}\right) .
$$

Note that replacing $\alpha$ by $-\alpha$ and adding/subtracting the two equations, we get

$$
\begin{aligned}
& \alpha\left(F_{\alpha}^{+}\right)=\alpha\left(a_{\alpha}^{+}\right), \\
& \alpha\left(F_{\alpha}^{-}\right)=\alpha\left(a_{\alpha}^{-}\right) .
\end{aligned}
$$

Using eqn (38), the first of these can be sharpened as follows:

$$
\alpha\left(F_{\alpha}^{+}\right)=0=\alpha\left(a_{\alpha}^{+}\right) .
$$

Next, inserting eqn (47) together with the functional equation (25) into the differential equation (41) for the Cartan part of the gauge potential, we see that this equation can be solved by setting

$$
A_{k}^{\mathfrak{h}}(q, u)=-\sum_{\alpha \in \Delta} \zeta(\alpha(q)) a_{k}^{-\alpha} F_{\alpha}-a_{k}^{\mathfrak{h}}(u),
$$

where the $a_{k}^{\mathfrak{h}}(u)$ are constants that must be determined from the remaining equations, provided we assume the coefficients $a_{k}^{\alpha}$ to satisfy the relation

$$
\sum_{\alpha \in \Delta} \alpha_{j} a_{k}^{-\alpha} F_{\alpha}=0 \quad \text { for } 1 \leqslant j, k \leqslant r .
$$

Converted into a tensor equation, it reads

$$
\sum_{\alpha \in \Delta} H_{\alpha} \otimes F_{\alpha} \otimes a_{-\alpha}=0,
$$


which leads back to eqn (66) by taking the scalar product with $H_{j}$ in the first and with $H_{k}$ in the third tensor factor. Note that in the degenerate case, the same argument works, but eqn (66/67) is not needed. Similarly, inserting eqn (47) together with the functional equation (29) into eqn (45), we obtain

$$
\begin{aligned}
& \alpha_{k}(-\Phi(\alpha(q), u) \Phi(\alpha(q),-v)+(\zeta(u)-\zeta(v)) \Phi(\alpha(q), u-v)) \\
& -\alpha\left(F_{-\alpha}\right) a_{k}^{\alpha} \Phi(\alpha(q), u) \Phi(\alpha(q),-v) \\
& -\Phi(\alpha(q), u-v)\left(\alpha\left(a_{k}^{\mathfrak{h}}(u)\right)-\alpha\left(a_{k}^{\mathfrak{h}}(v)\right)\right) \\
& -\alpha\left(a_{\alpha}\right) a_{k}^{-\alpha} \Phi(\alpha(q), u) \Phi(\alpha(q),-v)=0 .
\end{aligned}
$$

Obviously, the terms proportional to $\Phi(\alpha(q), u-v)$ cancel provided we set

$$
a_{k}^{\mathfrak{h}}(u)=\zeta(u) H_{k}
$$

and the remaining terms cancel if we impose the relation

$$
\alpha_{k}+\alpha\left(F_{-\alpha}\right) a_{k}^{\alpha}+\alpha\left(a_{\alpha}\right) a_{k}^{-\alpha}=0 \text {. }
$$

Converted to a vector equation in $\mathfrak{h}_{\mathbb{R}}$, it reads

$$
H_{\alpha}+\alpha\left(F_{-\alpha}\right) a_{\alpha}+\alpha\left(a_{\alpha}\right) a_{-\alpha}=0
$$

which leads back to eqn (69) by taking the scalar product with $H_{k}$. Even simpler to handle is eqn (46), which by insertion of the functional equation (28) reduces to the relation

$$
\begin{aligned}
& \alpha\left(F_{\beta}\right) a_{k}^{\alpha}-N_{\alpha, \beta} a_{k}^{\alpha+\beta}-\beta\left(a_{\alpha}\right) a_{k}^{\beta}=0 \\
& \quad \text { for } \alpha, \beta \in \Delta \text { such that } \beta \neq \pm \alpha .
\end{aligned}
$$

Converted to a vector equation in $\mathfrak{h}_{\mathbb{R}}$, it reads

$$
\begin{aligned}
& \alpha\left(F_{\beta}\right) a_{\alpha}-N_{\alpha, \beta} a_{\alpha+\beta}-\beta\left(a_{\alpha}\right) a_{\beta}=0 \\
& \text { for } \alpha, \beta \in \Delta \text { such that } \beta \neq \pm \alpha,
\end{aligned}
$$

which leads back to eqn (71) by taking the scalar product with $H_{k}$.

Before proceeding to the solution of the remaining equations, let us pause to draw a few consequences of the algebraic constraints (61)- 64 and $(69 / 70)$ derived so far; this will help us considerably to simplify our further work. First of all, eqns (61)- 64) state that

$$
\alpha\left(F_{-\alpha}\right)=-\alpha\left(F_{\alpha}\right)=-\alpha\left(a_{\alpha}\right)=\alpha\left(a_{-\alpha}\right),
$$

implying that eqn $(70)$ can be reduced to

$$
\alpha\left(a_{\alpha}\right)\left(a_{\alpha}-a_{-\alpha}\right)=H_{\alpha}
$$


Applying $\alpha$ to this relation and using the previous equation again, we conclude that

$$
\alpha\left(a_{\alpha}\right)=\epsilon_{\alpha} \frac{|\alpha|}{\sqrt{2}} \quad \text { and } \quad a_{\alpha}^{-}=\frac{\epsilon_{\alpha}}{\sqrt{2}|\alpha|} H_{\alpha}
$$

where $\epsilon_{\alpha}=\epsilon_{-\alpha}$ is a sign factor $( \pm 1)$. Next, we simplify all these equations by showing that eqns $(\sqrt{71} / / 72)$ and $(73)$ in fact force the vectors $a_{\alpha}$ and $F_{\alpha}$ to be equal. To prove this, we begin by symmetrizing eqn (72) with respect to the exchange of $\alpha$ and $\beta$, obtaining

$$
\alpha\left(F_{\beta}-a_{\beta}\right) a_{\alpha}+\beta\left(F_{\alpha}-a_{\alpha}\right) a_{\beta}=0 .
$$

Symmetrizing with respect to the exchange of $\beta$ and $-\beta$ and inserting eqn (73) gives

$$
\alpha\left(F_{\beta}^{+}-a_{\beta}^{+}\right) a_{\alpha}+\frac{\epsilon_{\beta}}{\sqrt{2}|\beta|} \beta\left(F_{\alpha}-a_{\alpha}\right) H_{\beta}=0 .
$$

Symmetrizing again with respect to the exchange of $\alpha$ and $-\alpha$ and inserting eqn (73) then leads to

$$
\frac{\epsilon_{\alpha}}{\sqrt{2}|\alpha|} \alpha\left(F_{\beta}^{+}-a_{\beta}^{+}\right) H_{\alpha}+\frac{\epsilon_{\beta}}{\sqrt{2}|\beta|} \beta\left(F_{\alpha}^{+}-a_{\alpha}^{+}\right) H_{\beta}=0
$$

But $H_{\alpha}$ and $H_{\beta}$ are linearly independent since, as stated in eqn (72), the roots $\alpha$ and $\beta$ are supposed to be non-proportional, so the coefficients must vanish separately, that is, for any two roots $\alpha, \beta \in \Delta$, we have

$$
\beta\left(F_{\alpha}^{+}-a_{\alpha}^{+}\right)=0
$$

whenever $\beta$ is not proportional to $\alpha$ and, according to eqn (64), also when $\beta$ is proportional to $\alpha$. Since $\Delta$ generates $\mathfrak{h}_{\mathbb{R}}$, this simply means that $a_{\alpha}^{+}=F_{\alpha}^{+}$. Inserting this conclusion back into eqn (74) and applying once more the same argument, we arrive at the result that $a_{\alpha}=F_{\alpha}$. With this result, eqns (61) $-(\sqrt{63})$ and (70) reduce to trivial identities whereas eqns (64), (73), (72), (67), (47) with $(\sqrt[49]{67}$ and $(\sqrt[65]{6})$ with (68) assume the form given in eqns (51), (52), (53), (54), (58) and (59), respectively.

Let us summarize the results obtained so far. With the exception of eqn (42), the system of equations (41)-(46) has been completely solved in terms of the explicit formulae (56) $-(59)$ for the gauge potential with the explicit formula (52) for the odd part $F_{\alpha}^{-}$of the coefficient vectors $F_{\alpha}$ and the algebraic constraints (51), (53) and (54). Thus we are left with the task of verifying the implications of eqns (14), (15) and (42).

Beginning with eqn (14), we use the functional equation (25) to compute

$$
\begin{aligned}
\partial_{k} A_{l}^{\mathfrak{h}}(q, u)-\partial_{l} A_{k}^{\mathfrak{h}}(q, u)+\sum_{\alpha \in \Delta} A_{k}^{\alpha}(q, u) A_{l}^{-\alpha}(q, u) H_{\alpha} \\
=\sum_{\alpha \in \Delta} \zeta^{\prime}(\alpha(q))\left(\alpha_{l}\left(H_{k}, F_{-\alpha}\right) F_{\alpha}-\alpha_{k}\left(H_{l}, F_{-\alpha}\right) F_{\alpha}\right) \\
\quad+\sum_{\alpha \in \Delta} \Phi(\alpha(q), u) \Phi(-\alpha(q), u)\left(H_{k}, F_{\alpha}\right)\left(H_{l}, F_{-\alpha}\right) H_{\alpha}
\end{aligned}
$$




$$
\begin{aligned}
& \begin{array}{r}
=\frac{1}{2} \sum_{\alpha \in \Delta} \zeta^{\prime}(\alpha(q))\left(+\alpha_{l}\left(H_{k}, F_{-\alpha}\right) F_{\alpha}-\alpha_{l}\left(H_{k}, F_{\alpha}\right) F_{-\alpha}\right. \\
-\alpha_{k}\left(H_{l}, F_{-\alpha}\right) F_{\alpha}+\alpha_{k}\left(H_{l}, F_{\alpha}\right) F_{-\alpha}
\end{array} \\
& \left.+\left(H_{k}, F_{\alpha}\right)\left(H_{l}, F_{-\alpha}\right) H_{\alpha}-\left(H_{k}, F_{-\alpha}\right)\left(H_{l}, F_{\alpha}\right) H_{\alpha}\right) \\
& -\zeta^{\prime}(u) \sum_{\alpha \in \Delta}\left(H_{k}, F_{\alpha}\right)\left(H_{l}, F_{-\alpha}\right) H_{\alpha} \\
& =\sum_{\alpha \in \Delta} \zeta^{\prime}(\alpha(q))\left(-\alpha_{l}\left(H_{k}, F_{\alpha}^{-}\right) F_{\alpha}^{+}+\alpha_{l}\left(H_{k}, F_{\alpha}^{+}\right) F_{\alpha}^{-}\right. \\
& +\alpha_{k}\left(H_{l}, F_{\alpha}^{-}\right) F_{\alpha}^{+}-\alpha_{k}\left(H_{l}, F_{\alpha}^{+}\right) F_{\alpha}^{-} \\
& \left.+\left(H_{k}, F_{\alpha}^{-}\right)\left(H_{l}, F_{\alpha}^{+}\right) H_{\alpha}-\left(H_{k}, F_{\alpha}^{+}\right)\left(H_{l}, F_{\alpha}^{-}\right) H_{\alpha}\right) \\
& -\zeta^{\prime}(u) \sum_{\alpha \in \Delta}\left(H_{k}, F_{\alpha}\right)\left(H_{l}, F_{-\alpha}\right) H_{\alpha}
\end{aligned}
$$

and can use eqns (52) and (54) to verify that the terms under the first sum cancel mutually in pairs whereas the second sum vanishes. Note that in the degenerate case, the same argument works, but eqn (54) is not needed.

For the proof of eqn (15), the trick is to split the sum over roots $\beta$ coming from the third and fourth term into contributions with $\beta=\alpha$, which cancel mutually, contributions with $\beta=-\alpha$, which combine with the contributions coming from the first and second term (transformed using the functional equation (301)), and the remaining contributions with $\beta \neq \pm \alpha$ : these can be complemented by terms that also cancel mutually (marked by underlining) and then be combined with the contributions from the fifth term (transformed using the functional equation (31)):

$$
\begin{aligned}
\partial_{k} A_{l}^{\alpha}(q, u)-\partial_{l} A_{k}^{\alpha}(q, u) & +\alpha\left(A_{k}^{\mathfrak{h}}(q, u)\right) A_{l}^{\alpha}(q, u)-\alpha\left(A_{l}^{\mathfrak{h}}(q, u)\right) A_{k}^{\alpha}(q, u) \\
& +\sum_{\substack{\beta, \gamma \in \Delta \\
\beta+\gamma=\alpha}} N_{\beta, \gamma} A_{k}^{\beta}(q, u) A_{l}^{\gamma}(q, u) \\
= & \Phi^{\prime}(\alpha(q), u) \alpha_{k}\left(H_{l}, F_{\alpha}\right)-\Phi^{\prime}(\alpha(q), u) \alpha_{l}\left(H_{k}, F_{\alpha}\right) \\
+ & \Phi(\alpha(q), u)\left(\sum_{\beta \in \Delta} \zeta(\beta(q)) \alpha\left(F_{-\beta}\right)\left(H_{k}, F_{\beta}\right)\left(H_{l}, F_{\alpha}\right)-\zeta(u) \alpha_{k}\left(H_{l}, F_{\alpha}\right)\right) \\
- & \Phi(\alpha(q), u)\left(\sum_{\beta \in \Delta} \zeta(\beta(q)) \alpha\left(F_{-\beta}\right)\left(H_{l}, F_{\beta}\right)\left(H_{k}, F_{\alpha}\right)-\zeta(u) \alpha_{l}\left(H_{k}, F_{\alpha}\right)\right) \\
+ & \sum_{\substack{\gamma, \delta \in \Delta \\
\gamma+\delta=\alpha}} N_{\gamma, \delta} \Phi(\gamma(q), u) \Phi(\delta(q), u)\left(H_{k}, F_{\gamma}\right)\left(H_{l}, F_{\delta}\right)
\end{aligned}
$$




$$
\begin{aligned}
& =\left(\Phi^{\prime}(\alpha(q), u)-\Phi(\alpha(q), u) \zeta(u)\right)\left(\left(H_{k}, H_{\alpha}\right)\left(H_{l}, F_{\alpha}\right)-\left(H_{l}, H_{\alpha}\right)\left(H_{k}, F_{\alpha}\right)\right) \\
& +\Phi(\alpha(q), u) \zeta(\alpha(q)) \\
& \times\left(+\alpha\left(F_{-\alpha}\right)\left(H_{k}, F_{\alpha}\right)\left(H_{l}, F_{\alpha}\right)-\alpha\left(F_{\alpha}\right)\left(H_{k}, F_{-\alpha}\right)\left(H_{l}, F_{\alpha}\right)\right. \\
& \left.-\alpha\left(F_{-\alpha}\right)\left(H_{l}, F_{\alpha}\right)\left(H_{k}, F_{\alpha}\right)+\alpha\left(F_{\alpha}\right)\left(H_{l}, F_{-\alpha}\right)\left(H_{k}, F_{\alpha}\right)\right) \\
& +\Phi(\alpha(q), u) \sum_{\substack{\beta \in \Delta \\
\beta \neq \pm \alpha}} \zeta(\beta(q))\left(H_{k}, F_{\beta}\right)\left(\alpha\left(F_{-\beta}\right)\left(H_{l}, F_{\alpha}\right)+\underline{\beta\left(F_{\alpha}\right)\left(H_{l}, F_{-\beta}\right)}\right) \\
& -\Phi(\alpha(q), u) \sum_{\substack{\beta \in \Delta \\
\beta \neq \pm \alpha}} \zeta(\beta(q))\left(H_{l}, F_{\beta}\right)\left(\alpha\left(F_{-\beta}\right)\left(H_{k}, F_{\alpha}\right)+\underline{\beta\left(F_{\alpha}\right)\left(H_{k}, F_{-\beta}\right)}\right) \\
& +\Phi(\alpha(q), u) \sum_{\substack{\gamma, \delta \in \Delta \\
\gamma+\delta=\alpha}}(\zeta(\gamma(q))+\zeta(\delta(q))) N_{\gamma, \delta}\left(H_{k}, F_{\gamma}\right)\left(H_{l}, F_{\delta}\right) \\
& -\Phi(\alpha(q), u)(\zeta(\alpha(q)+u)-\zeta(u)) \sum_{\substack{\gamma, \delta \in \Delta \\
\gamma+\delta=\alpha}} N_{\gamma, \delta}\left(H_{k}, F_{\gamma}\right)\left(H_{l}, F_{\delta}\right) \\
& =\Phi(\alpha(q), u)(\zeta(\alpha(q)+u)-\zeta(\alpha(q))-\zeta(u)) \\
& \times\left(\left(H_{k}, H_{\alpha}\right)\left(H_{l}, F_{\alpha}^{+}\right)-\left(H_{k}, F_{\alpha}^{+}\right)\left(H_{l}, H_{\alpha}\right)\right. \\
& \left.-2 \alpha\left(F_{\alpha}^{-}\right)\left(H_{k}, F_{\alpha}^{-}\right)\left(H_{l}, F_{\alpha}^{+}\right)+2 \alpha\left(F_{\alpha}^{-}\right)\left(H_{k}, F_{\alpha}^{+}\right)\left(H_{l}, F_{\alpha}^{-}\right)\right) \\
& +\Phi(\alpha(q), u)(\zeta(\alpha(q)+u)-\zeta(u)) \\
& \times\left(2 \alpha\left(F_{\alpha}^{-}\right)\left(H_{k}, F_{\alpha}^{-}\right)\left(H_{l}, F_{\alpha}^{+}\right)-2 \alpha\left(F_{\alpha}^{-}\right)\left(H_{k}, F_{\alpha}^{+}\right)\left(H_{l}, F_{\alpha}^{-}\right)\right. \\
& \left.-\sum_{\substack{\beta, \gamma \in \Delta \\
\beta+\gamma=\alpha}} N_{\beta, \gamma}\left(H_{k}, F_{\beta}\right)\left(H_{l}, F_{\gamma}\right)\right) \\
& \begin{aligned}
+\Phi(\alpha(q), u) \sum_{\substack{\beta \in \Delta \\
\beta \neq \pm \alpha}} \zeta(\beta(q))\left(H_{k}, F_{\beta}\right) & \times\left(H_{l}, \alpha\left(F_{-\beta}\right) F_{\alpha}+\beta\left(F_{\alpha}\right) F_{-\beta}+N_{\beta, \alpha-\beta} F_{\alpha-\beta}\right)
\end{aligned} \\
& \begin{aligned}
-\Phi(\alpha(q), u) \sum_{\substack{\beta \in \Delta \\
\beta \neq \pm \alpha}} \zeta(\beta(q))\left(H_{l}, F_{\beta}\right) & \times\left(H_{k}, \alpha\left(F_{-\beta}\right) F_{\alpha}+\beta\left(F_{\alpha}\right) F_{-\beta}-N_{\alpha-\beta, \beta} F_{\alpha-\beta}\right) .
\end{aligned}
\end{aligned}
$$

The last two terms vanish due to eqn (53), while the first term vanishes due to eqn (52). The same reasoning shows that the second term will vanish provided we impose the condition (55). Note that in the degenerate case, the same argument works, but eqn (55) is not needed. 
The proof of eqn (42) proceeds along similar lines, using the functional equations (29)-(31):

$$
\begin{aligned}
& \alpha_{k} \Phi^{\prime}(\alpha(q), v) F_{\alpha}+(\zeta(u-v)+\zeta(v)) A_{k}^{\alpha}(q, v) H_{\alpha}+\Phi(\alpha(q), v) \alpha\left(A_{k}^{\mathfrak{h}}(q, v)\right) F_{\alpha} \\
& -\sum_{\substack{\gamma, \delta \in \Delta \\
\gamma+\delta=\alpha}} N_{\gamma, \delta} \Phi(\gamma(q), v) A_{k}^{\delta}(q, v) F_{\gamma} \\
& \text { - } \Phi(-\alpha(q), u-v) A_{k}^{\alpha}(q, u) H_{\alpha}+\sum_{j=1}^{r} \alpha_{j} A_{k}^{\alpha}(q, v) A_{j}^{\mathfrak{h}}(q, u) \\
& =\Phi^{\prime}(\alpha(q), v) \alpha_{k} F_{\alpha}+(\zeta(u-v)+\zeta(v)) \Phi(\alpha(q), v)\left(H_{k}, F_{\alpha}\right) H_{\alpha} \\
& +\Phi(\alpha(q), v) \sum_{\beta \in \Delta} \zeta(\beta(q)) \alpha\left(F_{-\beta}\right)\left(H_{k}, F_{\beta}\right) F_{\alpha}-\Phi(\alpha(q), v) \zeta(v) \alpha_{k} F_{\alpha} \\
& -\sum_{\substack{\gamma, \delta \in \Delta \\
\gamma+\delta=\alpha}} N_{\gamma, \delta} \Phi(\gamma(q), v) \Phi(\delta(q), v)\left(H_{k}, F_{\delta}\right) F_{\gamma} \\
& \text { - } \Phi(-\alpha(q), u-v) \Phi(\alpha(q), u)\left(H_{k}, F_{\alpha}\right) H_{\alpha} \\
& \text { - } \Phi(\alpha(q), v) \sum_{j=1}^{r} \sum_{\beta \in \Delta} \zeta(\beta(q)) \alpha_{j}\left(H_{j}, F_{-\beta}\right)\left(H_{k}, F_{\alpha}\right) F_{\beta} \\
& \text { - } \Phi(\alpha(q), v) \zeta(u) \sum_{j=1}^{r} \alpha_{j}\left(H_{k}, F_{\alpha}\right) H_{j} \\
& =\left(\Phi^{\prime}(\alpha(q), v)-\Phi(\alpha(q), v) \zeta(v)\right)\left(H_{k}, H_{\alpha}\right) F_{\alpha} \\
& +((\zeta(u-v)+\zeta(v)-\zeta(u)) \Phi(\alpha(q), v) \\
& \text { - } \Phi(-\alpha(q), u-v) \Phi(\alpha(q), u))\left(H_{k}, F_{\alpha}\right) H_{\alpha} \\
& +\Phi(\alpha(q), v) \zeta(\alpha(q))\left(+\alpha\left(F_{-\alpha}\right)\left(H_{k}, F_{\alpha}\right) F_{\alpha}-\alpha\left(F_{\alpha}\right)\left(H_{k}, F_{-\alpha}\right) F_{\alpha}\right. \\
& \left.-\alpha\left(F_{-\alpha}\right)\left(H_{k}, F_{\alpha}\right) F_{\alpha}+\alpha\left(F_{\alpha}\right)\left(H_{k}, F_{\alpha}\right) F_{-\alpha}\right) \\
& +\Phi(\alpha(q), v) \sum_{\substack{\beta \in \Delta \\
\beta \neq \pm \alpha}} \zeta(\beta(q))\left(H_{k}, F_{\beta}\right)\left(\alpha\left(F_{-\beta}\right) F_{\alpha}+\underline{\beta\left(F_{\alpha}\right) F_{-\beta}}\right) \\
& -\Phi(\alpha(q), v) \sum_{\substack{\beta \in \Delta \\
\beta \neq \pm \alpha}} \zeta(\beta(q))\left(H_{k}, \alpha\left(F_{-\beta}\right) F_{\alpha}+\underline{\beta\left(F_{\alpha}\right) F_{-\beta}}\right) F_{\beta}
\end{aligned}
$$




$$
\begin{aligned}
& -\Phi(\alpha(q), v) \sum_{\substack{\gamma, \delta \in \Delta \\
\gamma+\delta=\alpha}}(\zeta(\gamma(q))+\zeta(\delta(q))) N_{\gamma, \delta}\left(H_{k}, F_{\delta}\right) F_{\gamma} \\
& +\Phi(\alpha(q), v)(\zeta(\alpha(q)+v)-\zeta(v)) \sum_{\substack{\gamma, \delta \in \Delta \\
\gamma+\delta=\alpha}} N_{\gamma, \delta}\left(H_{k}, F_{\delta}\right) F_{\gamma} \\
& =\Phi(\alpha(q), v)(\zeta(\alpha(q)+v)-\zeta(\alpha(q))-\zeta(v))\left(\left(H_{k}, H_{\alpha}\right) F_{\alpha}-\left(H_{k}, F_{\alpha}\right) H_{\alpha}\right) \\
& +\Phi(\alpha(q), v) \zeta(\alpha(q)) 2 \alpha\left(F_{\alpha}^{-}\right)\left(\left(H_{k}, F_{\alpha}^{-}\right) F_{\alpha}^{+}-\left(H_{k}, F_{\alpha}^{+}\right) F_{\alpha}^{-}\right) \\
& \begin{aligned}
+\Phi(\alpha(q), v) \sum_{\substack{\beta \in \Delta \\
\beta \neq \pm \alpha}} \zeta(\beta(q)) \\
\times\left(H_{k}, F_{\beta}\right)\left(\alpha\left(F_{-\beta}\right) F_{\alpha}+\beta\left(F_{\alpha}\right) F_{-\beta}-N_{\alpha-\beta, \beta} F_{\alpha-\beta}\right)
\end{aligned} \\
& \begin{aligned}
-\Phi(\alpha(q), v) \sum_{\substack{\beta \in \Delta \\
\beta \neq \pm \alpha}} \zeta(\beta(q)) & \times\left(H_{k}, \alpha\left(F_{-\beta}\right) F_{\alpha}+\beta\left(F_{\alpha}\right) F_{-\beta}+N_{\beta, \alpha-\beta} F_{\alpha-\beta}\right) F_{\beta}
\end{aligned} \\
& +\Phi(\alpha(q), v)(\zeta(\alpha(q)+v)-\zeta(v)) \sum_{\substack{\gamma, \delta \in \Delta \\
\gamma+\delta=\alpha}} N_{\gamma, \delta}\left(H_{k}, F_{\delta}\right) F_{\gamma} \\
& =\Phi(\alpha(q), v)(\zeta(\alpha(q)+v)-\zeta(v)) \\
& \times\left(\left(H_{k}, H_{\alpha}\right) F_{\alpha}-\left(H_{k}, F_{\alpha}\right) H_{\alpha}-\sum_{\substack{\beta, \gamma \in \Delta \\
\beta+\gamma=\alpha}} N_{\beta, \gamma}\left(H_{k}, F_{\beta}\right) F_{\gamma}\right),
\end{aligned}
$$

where in the last step, we have used eqns (53) and (52). Again, this expression will vanish provided we impose the condition (55). Note that in the degenerate case, the same argument works, but eqn (55) is not needed.

Having concluded the proof of Proposition 1, we pass to analyzing the implications of the algebraic constraints that we have derived. As it turns out, the conditions stated in Proposition 1 are sufficiently strong to allow for a complete classification of all possible solutions. As a by-product, we shall be able to reduce eqn (52) to the form given in eqn (60).

A first step in this direction is taken by observing that the signs $\epsilon_{\alpha}$ that appear in eqn (52) may without loss of generality be assumed to be independent of $\alpha$ :

$$
\epsilon_{\alpha}=\epsilon \quad \text { for all } \alpha \in \Delta \text {. }
$$

This freedom of choice follows from the possibility of performing a transformation that changes the signs of the root generators without modifying any of the relations between 
generators and structure constants used in the preceding calculations: it is given by

$$
\begin{gathered}
E_{\alpha} \longrightarrow E_{\alpha}^{\prime}=\epsilon \epsilon_{\alpha} E_{\alpha}, \\
H_{\alpha} \longrightarrow H_{\alpha}^{\prime}=H_{\alpha}, \\
N_{\alpha, \beta} \longrightarrow N_{\alpha, \beta}^{\prime}=\frac{\epsilon \epsilon_{\alpha} \epsilon_{\beta}}{\epsilon_{\alpha+\beta}} N_{\alpha, \beta}, \\
F_{\alpha} \longrightarrow F_{\alpha}^{\prime}=\epsilon \epsilon_{\alpha} F_{\alpha} .
\end{gathered}
$$

and, omitting the primes, brings eqn (52) into the form

$$
F_{\alpha}^{-}=\frac{\epsilon}{\sqrt{2}|\alpha|} H_{\alpha} .
$$

Next, let us write down the system obtained from eqn (53) upon replacing $\alpha$ by $-\alpha$ and $\beta$ by $-\beta$ :

$$
\begin{aligned}
\alpha\left(F_{\beta}\right) F_{\alpha}-\beta\left(F_{\alpha}\right) F_{\beta} & =N_{\alpha, \beta} F_{\alpha+\beta}, \\
\alpha\left(F_{-\beta}\right) F_{\alpha}+\beta\left(F_{\alpha}\right) F_{-\beta} & =N_{\alpha,-\beta} F_{\alpha-\beta}, \\
-\alpha\left(F_{\beta}\right) F_{-\alpha}-\beta\left(F_{-\alpha}\right) F_{\beta} & =N_{-\alpha, \beta} F_{-\alpha+\beta}, \\
-\alpha\left(F_{-\beta}\right) F_{-\alpha}+\beta\left(F_{-\alpha}\right) F_{-\beta} & =N_{-\alpha,-\beta} F_{-\alpha-\beta} .
\end{aligned}
$$

Adding these four equations gives

$$
\alpha\left(F_{\beta}^{+}\right) F_{\alpha}^{-}-\beta\left(F_{\alpha}^{+}\right) F_{\beta}^{-}=\frac{1}{2} N_{\alpha, \beta} F_{\alpha+\beta}^{-}+\frac{1}{2} N_{\alpha,-\beta} F_{\alpha-\beta}^{-} .
$$

Inserting eqn (52) and separating the coefficients of $H_{\alpha}$ and $H_{\beta}$, we conclude that

$$
\frac{1}{|\beta|} 2 \beta\left(F_{\alpha}^{+}\right)=\frac{1}{|\alpha-\beta|} N_{\alpha,-\beta}-\frac{1}{|\alpha+\beta|} N_{\alpha, \beta},
$$

plus the same equation with $\alpha$ and $\beta$ interchanged. It is to be noted that this derivation is only valid when $\beta \neq \pm \alpha$, as stated in eqn (53): this supplementary condition is also needed in order to guarantee that $H_{\alpha}$ and $H_{\beta}$ are linearly independent but can in fact be eliminated from eqn (78) since this formula is automatically satisfied when $\beta= \pm \alpha$. (Indeed, for $\beta= \pm \alpha$ the rhs is understood to vanish since $2 \alpha$ and 0 do not belong to the root system $\Delta$, whereas the lhs vanishes as a consequence of eqn (51).)

The algebraic equation (78) is identical with a special case of eqn (42) of Ref. [1], obtained by replacing the coupling constants $g_{\gamma}$ by $1 /|\gamma|$. As has been shown in Sect. 2.2 of Ref. [1], there is only one type of simple complex Lie algebra $\mathfrak{g}$ for which there exists a solution, namely those of the $A$-series. In particular, $\mathfrak{g}$ is simply laced, and all its roots have the same length, which according to our convention equals $\sqrt{2}$, and eqn (76) simplifies to the form given in eqn (60). Explicitly, if $\mathfrak{g}=\mathfrak{s l}(n, \mathbb{C})$ with $\mathfrak{h}_{\mathbb{R}}$ consisting of the real diagonal $(n \times n)$-matrices, we have $\Delta=\left\{\alpha_{a b} / 1 \leqslant a \neq b \leqslant n\right\}$ with $\alpha_{a b}(H)=H_{a a}-H_{b b}$ for $H \in \mathfrak{h}_{\mathbb{R}}$ and take $E_{\alpha_{a b}}=E_{a b}$ where $E_{a b}$ is the matrix whose 
entry in the $a^{\text {th }}$ row and $b^{\text {th }}$ column is 1 while all other entries are 0 ; then the structure constants $N_{a b, c d}=N_{\alpha_{a b}, \alpha_{c d}}$ are given by

$$
N_{a b, c d}=\delta_{b c}-\delta_{a d}
$$

and writing $F_{a b}^{ \pm}=F_{\alpha_{a b}}^{ \pm}$, we have

$$
F_{a b}^{+}=-\frac{1}{2}\left(E_{a a}+E_{b b}\right)+\frac{1}{n} \mathbf{1}_{n}
$$

and

$$
F_{a b}^{-}=\frac{\epsilon}{2}\left(E_{a a}-E_{b b}\right)
$$

implying that for $\epsilon=+1$,

$$
F_{a b}=-E_{b b}+\frac{1}{n} \mathbf{1}_{n}
$$

while for $\epsilon=-1$,

$$
F_{a b}=-E_{a a}+\frac{1}{n} \mathbf{1}_{n}
$$

It is then easy to check that $F$, as defined by eqns (80)-(83), satisfies all the conditions stated in Proposition 1. To see this, assume for simplicity that $\epsilon=+1$ (noting that the case $\epsilon=-1$ can be obtained from this one by replacing $F_{\alpha}$ by $F_{-\alpha}$, which does not affect the validity of any of the equations (53)-(55)). Then assuming, for example, that $\alpha=\alpha_{a b}$ and $\beta=\alpha_{c d}$, the lhs $\alpha_{a b}\left(F_{c d}\right) F_{a b}-\alpha_{c d}\left(F_{a b}\right) F_{c d}$ and rhs $N_{a b, c d} F_{\alpha_{a b}+\alpha_{c d}}$ of eqn (53) are both equal to

$$
\delta_{a d}\left(E_{b b}-\frac{1}{n} \mathbf{1}_{n}\right)-\delta_{b c}\left(E_{d d}-\frac{1}{n} \mathbf{1}_{n}\right)
$$

except when $a=d$ and $b=c(\beta=-\alpha)$, where the rhs is understood to vanish while the lhs does not. Similarly, the formula

$$
\sum_{a=1}^{n}\left(E_{a a}-\frac{1}{n} \mathbf{1}_{n}\right)=0
$$

allow us to verify eqns (54) and (55): the lhs of eqn (54) becomes

$$
\begin{aligned}
\sum_{1 \leqslant a \neq b \leqslant n}\left(E_{a a}-E_{b b}\right) \otimes\left(E_{b b}-\frac{1}{n} \mathbf{1}_{n}\right) \otimes\left(E_{a a}-\frac{1}{n} \mathbf{1}_{n}\right) \\
=-\sum_{a=1}^{n} E_{a a} \otimes\left(E_{a a}-\frac{1}{n} \mathbf{1}_{n}\right) \otimes\left(E_{a a}-\frac{1}{n} \mathbf{1}_{n}\right) \\
+\sum_{b=1}^{n} E_{b b} \otimes\left(E_{b b}-\frac{1}{n} \mathbf{1}_{n}\right) \otimes\left(E_{b b}-\frac{1}{n} \mathbf{1}_{n}\right)
\end{aligned}
$$


which vanishes as required, while that of eqn (55), for $\alpha=\alpha_{a b}$, becomes

$$
\begin{aligned}
\sum_{\substack{1 \leqslant c \leqslant n \\
c \neq a, c \neq b}}\left(F_{a c} \otimes F_{c b}-F_{c b} \otimes F_{a c}\right) \\
=\sum_{\substack{1 \leqslant c \leqslant n \\
c \neq a, c \neq b}}\left(E_{c c}-\frac{1}{n} \mathbf{1}_{n}\right) \otimes\left(E_{b b}-\frac{1}{n} \mathbf{1}_{n}\right)-\left(E_{b b}-\frac{1}{n} \mathbf{1}_{n}\right) \otimes\left(E_{c c}-\frac{1}{n} \mathbf{1}_{n}\right) \\
=-\left(E_{a a}+E_{b b}-\frac{2}{n} \mathbf{1}_{n}\right) \otimes\left(E_{b b}-\frac{1}{n} \mathbf{1}_{n}\right)+\left(E_{b b}-\frac{1}{n} \mathbf{1}_{n}\right) \otimes\left(E_{a a}+E_{b b}-\frac{2}{n} \mathbf{1}_{n}\right) \\
=-\left(E_{a a}-E_{b b}\right) \otimes\left(E_{b b}-\frac{1}{n} \mathbf{1}_{n}\right)+\left(E_{b b}-\frac{1}{n} \mathbf{1}_{n}\right) \otimes\left(E_{a a}-E_{b b}\right) \\
=H_{a b} \otimes F_{a b}-F_{a b} \otimes H_{a b},
\end{aligned}
$$

as required. In this way, we have rederived the main result of Refs [5] and [6], which states that the dynamical $R$-matrix of the integrable Calogero model associated with the root system of the simple Lie algebra $\mathfrak{g}=\mathfrak{s l}(n, \mathbb{C})$ of the $A$-series can be gauge transformed to a numerical $R$-matrix.

\section{Calogero Models for Symmetric Pairs}

According to Ref. [1], the standard Lax matrix $L$ and the dynamical $R$-matrix for the Calogero models associated with the root system $\Delta$ of a symmetric pair $(\mathfrak{g}, \theta)$ read

$$
\begin{gathered}
L=\sum_{j=1}^{r} p_{j} H_{j}+\sum_{\alpha \in \tilde{\Delta}} i g_{\alpha} w(\alpha(q)) E_{\alpha}, \\
R=\sum_{\alpha \in \tilde{\Delta}} w(\alpha(q)) K_{\alpha} \otimes E_{\alpha}+\frac{1}{2} \sum_{\alpha \in \tilde{\Delta}} \frac{w^{\prime}(\alpha(q))}{w(\alpha(q))}\left(E_{\alpha} \otimes E_{-\alpha}+E_{\theta \alpha} \otimes E_{-\alpha}\right),
\end{gathered}
$$

for the degenerate model and

$$
\begin{aligned}
L(u)=\sum_{j=1}^{r} p_{j} H_{j}+\sum_{\alpha \in \tilde{\Delta}} i g_{\alpha} \Phi(\alpha(q), u) E_{\alpha}, \\
R(u, v)=-\frac{1}{2} \sum_{j=1}^{r}(\zeta(u-v)+\zeta(u+v)) H_{j} \otimes H_{j} \\
-\frac{1}{2}(\zeta(u-v)-\zeta(u+v)+2 \zeta(v)) C_{\mathfrak{z}} \\
+\sum_{\alpha \in \tilde{\Delta}} \Phi(\alpha(q), v) K_{\alpha} \otimes E_{\alpha} \\
-\frac{1}{2} \sum_{\alpha \in \tilde{\Delta}}\left(\Phi(\alpha(q), u-v) E_{\alpha} \otimes E_{-\alpha}+\Phi(\alpha(q),-u-v) E_{\theta \alpha} \otimes E_{-\alpha}\right),
\end{aligned}
$$


for the elliptic model, where $\Delta=\Delta_{0} \cup \tilde{\Delta}$ with

$$
\begin{aligned}
& \Delta_{0}=\{\alpha \in \Delta / \theta \alpha=\alpha\}, \\
& \tilde{\Delta}=\{\alpha \in \Delta / \theta \alpha \neq \alpha\} .
\end{aligned}
$$

As has been shown in Ref. [1], integrability requires the generators $K_{\alpha} \in \mathfrak{i b}_{0}$ appearing in eqns (85) and (87) to satisfy the constraints (22). Moreover, writing

$$
K_{\alpha}^{ \pm}=\frac{1}{2}\left(K_{\alpha} \pm K_{-\alpha}\right),
$$

we also impose the condition

$$
\alpha\left(K_{\alpha}^{+}\right)=0 \quad \text { for } \alpha \in \tilde{\Delta} \text { such that } \theta \alpha-\alpha \notin \Delta,
$$

which follows from eqn (2) by setting $\beta=-\alpha$ when $g_{\alpha} \neq 0$ but turns out to be true in general, independent of this hypothesis.

Before proceeding with the calculations, we pause to note that the $R$-matrices given by eqns (85) and (87) have certain symmetry properties with respect to the automorphism $\theta$ that we want to be preserved under the gauge transformation to $R^{\prime}(u, v)$ : in the degenerate case, $R$ takes values in $\mathfrak{k} \otimes \mathfrak{m}$ whereas in the elliptic case, $R(u, v)$ is even under the action of $\theta \otimes 1$ and odd under the action of $1 \otimes \theta$ when these are combined with a change of sign in the corresponding spectral parameter. This can be achieved by imposing a restriction on the action of $\theta$ on $g$ or, equivalently, on the gauge potentials $A_{j}$ : in the degenerate case, $g$ should take values in $K$ and the $A_{j}$ should take values in $\mathfrak{k}$, whereas in the elliptic case, we require that

$$
\theta(g(-u))=g(u), \theta\left(A_{j}(-u)\right)=A_{j}(u)
$$

or in terms of the components of the gauge potentials in the expansion (13),

$$
\theta\left(A_{j}^{\mathfrak{h}}(-u)\right)=A_{j}^{\mathfrak{h}}(u), A_{j}^{\theta \alpha}(-u)=A_{j}^{\alpha}(u) .
$$

In order to compute the content of eqns (11) and (12), we further expand the Cartan part of the gauge potential according to

$$
A_{j}^{\mathfrak{h}}(u)=\sum_{k=1}^{r+s} A_{j}^{k}(u) H_{k} .
$$

Then inserting eqns (87), (13) and (92) into eqn (12), we obtain

$$
\begin{aligned}
0= & \sum_{\alpha \in \tilde{\Delta}} \alpha_{k} \Phi^{\prime}(\alpha(q), v) K_{\alpha} \otimes E_{\alpha} \\
& \quad-\frac{1}{2} \sum_{\alpha \in \tilde{\Delta}} \alpha_{k} \Phi^{\prime}(\alpha(q), u-v) E_{\alpha} \otimes E_{-\alpha}-\frac{1}{2} \sum_{\alpha \in \tilde{\Delta}} \alpha_{k} \Phi^{\prime}(\alpha(q),-u-v) E_{\theta \alpha} \otimes E_{-\alpha}
\end{aligned}
$$




$$
\begin{aligned}
& -\sum_{j=1}^{r} \partial_{j} A_{k}^{\mathfrak{h}}(q, u) \otimes H_{j}-\sum_{j=1}^{r} \sum_{\alpha \in \Delta} \partial_{j} A_{k}^{\alpha}(q, u) E_{\alpha} \otimes H_{j} \\
& +\frac{1}{2} \sum_{j=1}^{r} \sum_{\alpha \in \Delta}(\zeta(u-v)+\zeta(u+v)) A_{k}^{\alpha}(q, u)\left[H_{j} \otimes H_{j}, E_{\alpha} \otimes 1\right] \\
& +\frac{1}{2} \sum_{j=1}^{r} \sum_{\alpha \in \Delta}(\zeta(u-v)+\zeta(u+v)) A_{k}^{\alpha}(q, v)\left[H_{j} \otimes H_{j}, 1 \otimes E_{\alpha}\right] \\
& +\frac{1}{2} \sum_{j=1}^{r+s}(\zeta(u-v)-\zeta(u+v)+2 \zeta(v)) A_{k}^{j}(q, u)\left[C_{\mathfrak{z}}, H_{j} \otimes 1\right] \\
& +\frac{1}{2} \sum_{j=1}^{r+s}(\zeta(u-v)-\zeta(u+v)+2 \zeta(v)) A_{k}^{j}(q, v)\left[C_{\mathfrak{z}}, 1 \otimes H_{j}\right] \\
& +\frac{1}{2} \sum_{\alpha \in \Delta}(\zeta(u-v)-\zeta(u+v)+2 \zeta(v)) A_{k}^{\alpha}(q, u)\left[C_{\mathfrak{z}}, E_{\alpha} \otimes 1\right] \\
& +\frac{1}{2} \sum_{j=1} \sum_{\gamma \in \Delta}(\zeta(u-v)-\zeta(u+v)+2 \zeta(v)) A_{k}^{\alpha}(q, v)\left[C_{\mathfrak{z}}, 1 \otimes E_{\alpha}\right] \\
& +\frac{1}{2} \sum_{j=1}^{r+s} \sum_{\gamma \in \tilde{\Delta}} \Phi(\gamma(q), u-v) A_{k}^{j}(q, v)\left[E_{\gamma} \otimes E_{-\gamma}, 1 \otimes H_{j}\right] \\
& +\sum_{j=\tilde{\Delta}}^{r+s} \sum_{j=1} \sum_{\gamma \in \tilde{\Delta}} \Phi(\gamma(q), v) A_{k}^{j}(q, u)\left[K_{\gamma} \otimes E_{\gamma}, H_{j} \otimes 1\right] \\
& +\sum_{\delta \in \Delta}^{r+s} \sum_{j=1}^{r+s} \Phi(\gamma(q), v) A_{k}^{\delta}(q, v)\left[K_{\gamma} \otimes E_{\gamma}, 1 \otimes E_{\delta}\right] \\
& +\sum_{\gamma \in \tilde{\Delta}} \Phi(\gamma(q), v) A_{k}^{\delta}(q, u)\left[K_{\gamma} \otimes E_{\gamma}, E_{\delta} \otimes 1\right] \\
& +\sum_{k}(q, v)\left[K_{\gamma} \otimes E_{\gamma}, 1 \otimes H_{j}\right]
\end{aligned}
$$




$$
\begin{aligned}
& +\frac{1}{2} \sum_{\substack{\gamma \in \tilde{\Delta} \\
\delta \in \Delta}} \Phi(\gamma(q), u-v) A_{k}^{\delta}(q, u)\left[E_{\gamma} \otimes E_{-\gamma}, E_{\delta} \otimes 1\right] \\
& +\frac{1}{2} \sum_{\substack{\gamma \in \tilde{\Delta} \\
\delta \in \Delta}} \Phi(\gamma(q), u-v) A_{k}^{\delta}(q, v)\left[E_{\gamma} \otimes E_{-\gamma}, 1 \otimes E_{\delta}\right] \\
& +\frac{1}{2} \sum_{\substack{\gamma \in \tilde{\Delta} \\
\delta \in \Delta}} \Phi(\gamma(q),-u-v) A_{k}^{\delta}(q, u)\left[E_{\theta \gamma} \otimes E_{-\gamma}, E_{\delta} \otimes 1\right] \\
& +\frac{1}{2} \sum_{\substack{\gamma \in \tilde{\Delta} \\
\delta \in \Delta}} \Phi(\gamma(q),-u-v) A_{k}^{\delta}(q, v)\left[E_{\theta \gamma} \otimes E_{-\gamma}, 1 \otimes E_{\delta}\right] \\
& +\sum_{j=1}^{r} \sum_{\alpha \in \Delta} \alpha_{j} A_{k}^{\alpha}(q, v) A_{j}^{\mathfrak{h}}(q, u) \otimes E_{\alpha}+\sum_{j=1}^{r} \sum_{\alpha, \beta \in \Delta} \beta_{j} A_{j}^{\alpha}(q, u) A_{k}^{\beta}(q, v) E_{\alpha} \otimes E_{\beta} .
\end{aligned}
$$

Using the definition of $C_{\mathfrak{z}}$,

$$
C_{\mathfrak{z}}=\sum_{j=r+1}^{r+s} H_{j} \otimes H_{j}+\sum_{\alpha \in \Delta_{0}} E_{\alpha} \otimes E_{-\alpha}
$$

and eqns (16)-(20) to carry out the commutators, together with the relation

$$
\sum_{j=1}^{r+s} \alpha_{j} H_{j}=H_{\alpha}, \quad \sum_{j=1}^{r} \alpha_{j} H_{j}=\left(H_{\alpha}\right)_{\mathfrak{a}}, \quad \sum_{j=r+1}^{r+s} \alpha_{j} H_{j}=\left(H_{\alpha}\right)_{\mathfrak{b}}
$$

we arrive at

$$
\begin{aligned}
0= & \sum_{\alpha \in \tilde{\Delta}} \alpha_{k} \Phi^{\prime}(\alpha(q), v) K_{\alpha} \otimes E_{\alpha} \\
& -\frac{1}{2} \sum_{\alpha \in \tilde{\Delta}} \alpha_{k} \Phi^{\prime}(\alpha(q), u-v) E_{\alpha} \otimes E_{-\alpha}-\frac{1}{2} \sum_{\alpha \in \tilde{\Delta}} \alpha_{k} \Phi^{\prime}(\alpha(q),-u-v) \theta E_{\alpha} \otimes E_{-\alpha} \\
& -\sum_{j=1}^{r} \partial_{j} A_{k}^{\mathfrak{h}}(q, u) \otimes H_{j}-\sum_{j=1}^{r} \sum_{\alpha \in \Delta} \partial_{j} A_{k}^{\alpha}(q, u) E_{\alpha} \otimes H_{j} \\
& +\frac{1}{2} \sum_{j=1}^{r} \sum_{\alpha \in \tilde{\Delta}} \alpha_{j}(\zeta(u-v)+\zeta(u+v)) A_{k}^{\alpha}(q, u) E_{\alpha} \otimes H_{j} \\
& +\frac{1}{2} \sum_{j=1}^{r} \sum_{\alpha \in \tilde{\Delta}} \alpha_{j}(\zeta(u-v)+\zeta(u+v)) A_{k}^{\alpha}(q, v) H_{j} \otimes E_{\alpha} \\
& -\frac{1}{2} \sum_{j=1}^{r+s} \sum_{\alpha \in \Delta_{0}} \alpha_{j}(\zeta(u-v)-\zeta(u+v)+2 \zeta(v)) A_{k}^{j}(q, u) E_{\alpha} \otimes E_{-\alpha}
\end{aligned}
$$




$$
\begin{aligned}
& +\frac{1}{2} \sum_{j=1}^{r+s} \sum_{\alpha \in \Delta_{0}} \alpha_{j}(\zeta(u-v)-\zeta(u+v)+2 \zeta(v)) A_{k}^{j}(q, v) E_{\alpha} \otimes E_{-\alpha} \\
& +\frac{1}{2} \sum_{j=r+1}^{r+s} \sum_{\alpha \in \Delta} \alpha_{j}(\zeta(u-v)-\zeta(u+v)+2 \zeta(v)) A_{k}^{\alpha}(q, u) E_{\alpha} \otimes H_{j} \\
& +\frac{1}{2} \sum_{j=r+1}^{r+s} \sum_{\alpha \in \Delta} \alpha_{j}(\zeta(u-v)-\zeta(u+v)+2 \zeta(v)) A_{k}^{\alpha}(q, v) H_{j} \otimes E_{\alpha} \\
& +\frac{1}{2} \sum_{\gamma \in \Delta_{0}}(\zeta(u-v)-\zeta(u+v)+2 \zeta(v)) A_{k}^{-\gamma}(q, u) H_{\gamma} \otimes E_{-\gamma} \\
& +\frac{1}{2} \sum_{\gamma \in \Delta_{0}}(\zeta(u-v)-\zeta(u+v)+2 \zeta(v)) A_{k}^{\gamma}(q, v) E_{\gamma} \otimes H_{-\gamma} \\
& +\frac{1}{2} \sum_{\substack{\alpha \in \Delta, \gamma \in \Delta_{0} \\
\alpha+\gamma \in \Delta}} N_{\gamma, \alpha}(\zeta(u-v)-\zeta(u+v)+2 \zeta(v)) A_{k}^{\alpha}(q, u) E_{\gamma+\alpha} \otimes E_{-\gamma} \\
& +\frac{1}{2} \sum_{\substack{\alpha \in \Delta, \gamma \in \Delta_{0} \\
\alpha-\gamma \in \Delta}} N_{-\gamma, \alpha}(\zeta(u-v)-\zeta(u+v)+2 \zeta(v)) A_{k}^{\alpha}(q, v) E_{\gamma} \otimes E_{-\gamma+\alpha} \\
& +\sum_{j=1}^{r+s} \sum_{\gamma \in \tilde{\Delta}} \gamma_{j} \Phi(\gamma(q), v) A_{k}^{j}(q, v) K_{\gamma} \otimes E_{\gamma} \\
& -\sum_{\substack{\gamma \in \tilde{\Delta} \\
\delta \in \Delta}} \delta\left(K_{\gamma}\right) \Phi(\gamma(q), v) A_{k}^{\delta}(q, u) E_{\delta} \otimes E_{\gamma} \\
& -\sum_{\gamma \in \tilde{\Delta}} \Phi(\gamma(q), v) A_{k}^{-\gamma}(q, v) K_{\gamma} \otimes H_{\gamma} \\
& -\sum_{\substack{\gamma \in \tilde{\Delta}, \delta \in \Delta \\
\gamma+\delta \in \Delta}} N_{\gamma, \delta} \Phi(\gamma(q), v) A_{k}^{\delta}(q, v) K_{\gamma} \otimes E_{\gamma+\delta} \\
& -\frac{1}{2} \sum_{j=1}^{r+s} \sum_{\gamma \in \tilde{\Delta}} \gamma_{j} \Phi(\gamma(q), u-v) A_{k}^{j}(q, u) E_{\gamma} \otimes E_{-\gamma} \\
& +\frac{1}{2} \sum_{j=1}^{r+s} \sum_{\gamma \in \tilde{\Delta}} \gamma_{j} \Phi(\gamma(q), u-v) A_{k}^{j}(q, v) E_{\gamma} \otimes E_{-\gamma} \\
& -\frac{1}{2} \sum_{j=1}^{r+s} \sum_{\gamma \in \tilde{\Delta}}(\theta \gamma)_{j} \Phi(\gamma(q),-u-v) A_{k}^{j}(q, u) \theta E_{\gamma} \otimes E_{-\gamma} \\
& +\frac{1}{2} \sum_{j=1}^{r+s} \sum_{\gamma \in \tilde{\Delta}} \gamma_{j} \Phi(\gamma(q),-u-v) A_{k}^{j}(q, v) \theta E_{\gamma} \otimes E_{-\gamma}
\end{aligned}
$$




$$
\begin{aligned}
& +\frac{1}{2} \sum_{\gamma \in \tilde{\Delta}} \Phi(\gamma(q), u-v) A_{k}^{-\gamma}(q, u) H_{\gamma} \otimes E_{-\gamma} \\
& +\frac{1}{2} \sum_{\substack{\gamma \in \tilde{\Delta}, \delta \in \Delta \\
\gamma+\delta \in \Delta}} N_{\gamma, \delta} \Phi(\gamma(q), u-v) A_{k}^{\delta}(q, u) E_{\gamma+\delta} \otimes E_{-\gamma} \\
& +\frac{1}{2} \sum_{\gamma \in \tilde{\Delta}} \Phi(\gamma(q), u-v) A_{k}^{\gamma}(q, v) E_{\gamma} \otimes H_{-\gamma} \\
& +\frac{1}{2} \sum_{\substack{\gamma \in \tilde{\Delta}, \delta \in \Delta \\
\gamma-\delta \in \Delta}} N_{-\gamma, \delta} \Phi(\gamma(q), u-v) A_{k}^{\delta}(q, v) E_{\gamma} \otimes E_{-\gamma+\delta} \\
& +\frac{1}{2} \sum_{\substack{\gamma \in \tilde{\Delta} \\
+}(\gamma(q),-u-v) A_{k}^{-\theta \gamma}(q, u) \theta H_{\gamma} \otimes E_{-\gamma}} \sum_{\substack{\gamma \in \tilde{\Delta}, \delta \in \Delta \\
\theta \gamma+\delta \in \Delta}} N_{\theta \gamma, \delta} \Phi(\gamma(q),-u-v) A_{k}^{\delta}(q, u) E_{\theta \gamma+\delta} \otimes E_{-\gamma} \\
& +\frac{1}{2} \sum_{\gamma \in \tilde{\Delta}} \Phi(\gamma(q),-u-v) A_{k}^{\gamma}(q, v) E_{\theta \gamma} \otimes H_{-\gamma} \\
& +\frac{1}{2} \sum_{\substack{\gamma \in \tilde{\Delta}, \delta \in \Delta \\
\gamma-\delta \in \Delta}}^{r} N_{-\gamma, \delta} \Phi(\gamma(q),-u-v) A_{k}^{\delta}(q, v) E_{\theta \gamma} \otimes E_{-\gamma+\delta} \\
& +\sum_{j=1}^{r} \sum_{\substack{\alpha \in \Delta \\
\alpha}} A_{j}^{\alpha}(q, v) A_{j}^{\mathfrak{h}}(q, u) \otimes E_{\alpha}+\sum_{j=1}^{r} \sum_{\alpha, \beta \in \Delta} \beta_{j} A_{j}^{\alpha}(q, u) A_{k}^{\beta}(q, v) E_{\alpha} \otimes E_{\beta}
\end{aligned}
$$

Relabelling summation indices and using cyclicity and antisymmetry of the structure constants, we can bring this expression into the following form:

$$
\begin{aligned}
0= & \sum_{\alpha \in \tilde{\Delta}} \alpha_{k} \Phi^{\prime}(\alpha(q), v) K_{\alpha} \otimes E_{\alpha} \\
& -\frac{1}{2} \sum_{\alpha \in \tilde{\Delta}} \alpha_{k} \Phi^{\prime}(\alpha(q), u-v) E_{\alpha} \otimes E_{-\alpha}-\frac{1}{2} \sum_{\alpha \in \tilde{\Delta}} \alpha_{k} \Phi^{\prime}(\alpha(q),-u-v) \theta E_{\alpha} \otimes E_{-\alpha} \\
& -\sum_{j=1}^{r} \partial_{j} A_{k}^{\mathfrak{h}}(q, u) \otimes H_{j}-\sum_{j=1}^{r} \sum_{\alpha \in \Delta} \partial_{j} A_{k}^{\alpha}(q, u) E_{\alpha} \otimes H_{j} \\
& +\frac{1}{2} \sum_{j=1}^{r} \sum_{\alpha \in \tilde{\Delta}} \alpha_{j}(\zeta(u-v)+\zeta(u+v)) A_{k}^{\alpha}(q, u) E_{\alpha} \otimes H_{j} \\
& +\frac{1}{2} \sum_{j=1}^{r} \sum_{\alpha \in \tilde{\Delta}} \alpha_{j}(\zeta(u-v)+\zeta(u+v)) A_{k}^{\alpha}(q, v) H_{j} \otimes E_{\alpha} \\
& -\frac{1}{2} \sum_{j=1}^{r+s} \sum_{\alpha \in \Delta_{0}} \alpha_{j}(\zeta(u-v)-\zeta(u+v)+2 \zeta(v)) A_{k}^{j}(q, u) E_{\alpha} \otimes E_{-\alpha}
\end{aligned}
$$




$$
\begin{aligned}
& +\frac{1}{2} \sum_{j=1}^{r+s} \sum_{\alpha \in \Delta_{0}} \alpha_{j}(\zeta(u-v)-\zeta(u+v)+2 \zeta(v)) A_{k}^{j}(q, v) E_{\alpha} \otimes E_{-\alpha} \\
& +\frac{1}{2} \sum_{j=r+1}^{r+s} \sum_{\alpha \in \Delta} \alpha_{j}(\zeta(u-v)-\zeta(u+v)+2 \zeta(v)) A_{k}^{\alpha}(q, u) E_{\alpha} \otimes H_{j} \\
& +\frac{1}{2} \sum_{j=r+1}^{r+s} \sum_{\alpha \in \Delta} \alpha_{j}(\zeta(u-v)-\zeta(u+v)+2 \zeta(v)) A_{k}^{\alpha}(q, v) H_{j} \otimes E_{\alpha} \\
& -\frac{1}{2} \sum_{\alpha \in \Delta_{0}}(\zeta(u-v)-\zeta(u+v)+2 \zeta(v)) A_{k}^{\alpha}(q, u) H_{\alpha} \otimes E_{\alpha} \\
& -\frac{1}{2} \sum_{\alpha \in \Delta_{0}}(\zeta(u-v)-\zeta(u+v)+2 \zeta(v)) A_{k}^{\alpha}(q, v) E_{\alpha} \otimes H_{\alpha} \\
& -\frac{1}{2} \sum_{\substack{\alpha \in \Delta, \beta \in \Delta_{0} \\
\alpha+\beta \in \Delta}} N_{\alpha, \beta}(\zeta(u-v)-\zeta(u+v)+2 \zeta(v)) A_{k}^{\alpha+\beta}(q, u) E_{\alpha} \otimes E_{\beta} \\
& +\frac{1}{2} \sum_{\substack{\alpha \in \Delta_{0}, \beta \in \Delta \\
\alpha+\beta \in \Delta}} N_{\alpha, \beta}(\zeta(u-v)-\zeta(u+v)+2 \zeta(v)) A_{k}^{\alpha+\beta}(q, v) E_{\alpha} \otimes E_{\beta} \\
& +\sum_{j=1}^{r+s} \sum_{\alpha \in \tilde{\Delta}} \alpha_{j} \Phi(\alpha(q), v) A_{k}^{j}(q, v) K_{\alpha} \otimes E_{\alpha} \\
& -\sum_{\substack{\alpha \in \Delta \\
\beta \in \tilde{\Delta}}} \alpha\left(K_{\beta}\right) \Phi(\beta(q), v) A_{k}^{\alpha}(q, u) E_{\alpha} \otimes E_{\beta} \\
& -\sum_{\alpha \in \tilde{\Delta}} \Phi(\alpha(q), v) A_{k}^{-\alpha}(q, v) K_{\alpha} \otimes H_{\alpha} \\
& -\sum_{\substack{\gamma \in \tilde{\Delta}, \delta \in \Delta \\
\gamma+\delta \in \Delta}} N_{\gamma, \delta} \Phi(\gamma(q), v) A_{k}^{\delta}(q, v) K_{\gamma} \otimes E_{\gamma+\delta} \\
& -\frac{1}{2} \sum_{j=1}^{r+s} \sum_{\alpha \in \tilde{\Delta}} \alpha_{j} \Phi(\alpha(q), u-v) A_{k}^{j}(q, u) E_{\alpha} \otimes E_{-\alpha} \\
& +\frac{1}{2} \sum_{j=1}^{r+s} \sum_{\alpha \in \tilde{\Delta}} \alpha_{j} \Phi(\alpha(q), u-v) A_{k}^{j}(q, v) E_{\alpha} \otimes E_{-\alpha} \\
& -\frac{1}{2} \sum_{j=1}^{r+s} \sum_{\alpha \in \tilde{\Delta}}(\theta \alpha)_{j} \Phi(\alpha(q),-u-v) A_{k}^{j}(q, u) \theta E_{\alpha} \otimes E_{-\alpha} \\
& +\frac{1}{2} \sum_{j=1}^{r+s} \sum_{\alpha \in \tilde{\Delta}} \alpha_{j} \Phi(\alpha(q),-u-v) A_{k}^{j}(q, v) \theta E_{\alpha} \otimes E_{-\alpha}
\end{aligned}
$$




$$
\begin{aligned}
& -\frac{1}{2} \sum_{\alpha \in \tilde{\Delta}} \Phi(-\alpha(q), u-v) A_{k}^{\alpha}(q, u) H_{\alpha} \otimes E_{\alpha} \\
& -\frac{1}{2} \sum_{\substack{\alpha \in \Delta, \beta \in \tilde{\Delta} \\
\alpha+\beta \in \Delta}} N_{\alpha, \beta} \Phi(-\beta(q), u-v) A_{k}^{\alpha+\beta}(q, u) E_{\alpha} \otimes E_{\beta} \\
& -\frac{1}{2} \sum_{\alpha \in \tilde{\Delta}} \Phi(\alpha(q), u-v) A_{k}^{\alpha}(q, v) E_{\alpha} \otimes H_{\alpha} \\
& +\frac{1}{2} \sum_{\substack{\alpha \in \tilde{\Delta}, \beta \in \Delta \\
\alpha+\beta \in \Delta}} N_{\alpha, \beta} \Phi(\alpha(q), u-v) A_{k}^{\alpha+\beta}(q, v) E_{\alpha} \otimes E_{\beta} \\
& -\frac{1}{2} \sum_{\substack{\alpha \in \tilde{\Delta} \\
-}} \sum_{\substack{\alpha \in \Delta, \beta \in \tilde{\Delta} \\
\alpha+\beta \in \Delta}} N_{\alpha, \beta} \Phi(-\beta(q),-u-v) A_{k}^{\alpha+\beta}(q,-u) \theta E_{\alpha} \otimes E_{\beta} \\
& -\frac{1}{2} \sum_{\substack{\alpha \in \tilde{\Delta} \\
+}} \Phi(-\alpha(q),-u-v) A_{k}^{\alpha}(q,-v) E_{\alpha} \otimes \theta H_{\alpha} \\
& +\frac{1}{2} \sum_{\substack{\alpha \in \tilde{\Delta}, \beta \in \Delta \\
\alpha+\beta \in \Delta}}^{r} N_{\alpha, \beta} \Phi(\alpha(q),-u-v) A_{k}^{\alpha+\beta}(q, v) \theta E_{\alpha} \otimes E_{\beta} \\
& +\sum_{j=1}^{r} \sum_{\alpha \in \Delta} \alpha_{j} A_{k}^{\alpha}(q, v) A_{j}^{\mathfrak{h}}(q, u) \otimes E_{\alpha}+\sum_{j=1}^{r} \sum_{\alpha, \beta \in \Delta} \beta_{j} A_{j}^{\alpha}(q, u) A_{k}^{\beta}(q, v) E_{\alpha} \otimes E_{\beta} .
\end{aligned}
$$

Noting that for $\alpha \in \Delta_{0}$, we have $\alpha_{j}=0$ for $1 \leqslant j \leqslant r$ and $\left(H_{\alpha}\right)_{\mathfrak{a}}=0$, we can now identify the components of eqn (12) along the various subspaces of $\mathfrak{g} \otimes \mathfrak{g}$ :

- The components along $\mathfrak{h} \otimes H_{j}$ lead to the following system of equations.

For $1 \leqslant j \leqslant r:$

$$
\partial_{j} A_{k}^{\mathfrak{h}}(q, u)+\sum_{\alpha \in \tilde{\Delta}} \alpha_{j} \Phi(\alpha(q), v) A_{k}^{-\alpha}(q, v) K_{\alpha}=0 .
$$

For $r+1 \leqslant j \leqslant r+s$ :

$$
\sum_{\alpha \in \tilde{\Delta}} \alpha_{j} \Phi(\alpha(q), v) A_{k}^{-\alpha}(q, v) K_{\alpha}=0
$$


- The components along $\mathfrak{h} \otimes \mathfrak{g}_{\alpha}$ lead to the following system of equations. For $\alpha \in \tilde{\Delta}$ :

$$
\begin{aligned}
& \alpha_{k} \Phi^{\prime}(\alpha(q), v) K_{\alpha}+\frac{1}{2}(\zeta(u-v)+\zeta(u+v)) A_{k}^{\alpha}(q, v)\left(H_{\alpha}\right)_{\mathfrak{a}} \\
& +\frac{1}{2}(\zeta(u-v)-\zeta(u+v)+2 \zeta(v)) A_{k}^{\alpha}(q, v)\left(H_{\alpha}\right)_{\mathfrak{b}} \\
& +\Phi(\alpha(q), v) \alpha\left(A_{k}^{\mathfrak{h}}(q, v)\right) K_{\alpha}-\sum_{\substack{\gamma \in \tilde{\Delta}, \delta \in \Delta \\
\gamma+\delta=\alpha}} N_{\gamma, \delta} \Phi(\gamma(q), v) A_{k}^{\delta}(q, v) K_{\gamma} \\
& +\frac{1}{2} \Phi(\alpha(q), v-u) A_{k}^{\alpha}(q, u) H_{\alpha}+\frac{1}{2} \Phi(\alpha(q), v+u) A_{k}^{\alpha}(q,-u) \theta H_{\alpha} \\
& +\sum_{j=1}^{r} \alpha_{j} A_{k}^{\alpha}(q, v) A_{j}^{\mathfrak{h}}(q, u)=0 \text {. }
\end{aligned}
$$

For $\alpha \in \Delta_{0}$ :

$$
\begin{aligned}
& \frac{1}{2}(\zeta(u-v)-\zeta(u+v)+2 \zeta(v))\left(A_{k}^{\alpha}(q, v)-A_{k}^{\alpha}(q, u)\right) H_{\alpha} \\
& \quad-\sum_{\substack{\gamma, \delta \in \tilde{\Delta} \\
\gamma+\delta=\alpha}} N_{\gamma, \delta} \Phi(\gamma(q), v) A_{k}^{\delta}(q, v) K_{\gamma}=0 .
\end{aligned}
$$

- The components along $\mathfrak{g}_{\alpha} \otimes H_{j}$ lead to the following system of equations.

For $\alpha \in \tilde{\Delta}, 1 \leqslant j \leqslant r$ :

$$
\begin{aligned}
& \partial_{j} A_{k}^{\alpha}(q, u)-\frac{1}{2} \alpha_{j}(\zeta(u-v)+\zeta(u+v)) A_{k}^{\alpha}(q, u) \\
& +\frac{1}{2} \alpha_{j}\left(\Phi(\alpha(q), u-v) A_{k}^{\alpha}(q, v)+\Phi(\alpha(q), u+v) A_{k}^{\alpha}(q,-v)\right)=0
\end{aligned}
$$

For $\alpha \in \Delta_{0}, 1 \leqslant j \leqslant r:$

$$
\partial_{j} A_{k}^{\alpha}(q, u)=0
$$

For $\alpha \in \tilde{\Delta}, r+1 \leqslant j \leqslant r+s$ :

$$
\begin{aligned}
& (\zeta(u-v)-\zeta(u+v)+2 \zeta(v)) A_{k}^{\alpha}(q, u) \\
& -\left(\Phi(\alpha(q), u-v) A_{k}^{\alpha}(q, v)-\Phi(\alpha(q), u+v) A_{k}^{\alpha}(q,-v)\right)=0 .
\end{aligned}
$$

For $\alpha \in \Delta_{0}, r+1 \leqslant j \leqslant r+s$ :

$$
A_{k}^{\alpha}(q, u)-A_{k}^{\alpha}(q, v)=0 .
$$


- The components along $\mathfrak{g}_{\alpha} \otimes \mathfrak{g}_{\beta}$ lead to the following system of equations.

For $\alpha \in \Delta_{0}$ and $\beta=-\alpha$ :

$$
\alpha\left(A_{k}^{\mathfrak{h}}(q, u)-A_{k}^{\mathfrak{h}}(q, v)\right)=0 .
$$

For $\alpha, \beta \in \Delta_{0}$ with $\alpha+\beta \neq 0$, no new condition arises, due to eqn (102).

For $\alpha \in \Delta_{0}, \beta \in \tilde{\Delta}$ :

$$
\begin{aligned}
& \frac{1}{2} N_{\alpha, \beta}(\zeta(u-v)-\zeta(u+v)+2 \zeta(v)) A_{k}^{\alpha+\beta}(q, v) \\
& -\alpha\left(K_{\beta}\right) \Phi(\beta(q), v) A_{k}^{\alpha}(q, u) \\
& +\frac{1}{2} N_{\alpha, \beta}\left(\Phi(\beta(q), v-u) A_{k}^{\alpha+\beta}(q, u)+\Phi(\beta(q), v+u) A_{k}^{\alpha+\beta}(q,-u)\right) \\
& +\sum_{j=1}^{r} \beta_{j} A_{j}^{\alpha}(q, u) A_{k}^{\beta}(q, v)=0 .
\end{aligned}
$$

For $\alpha \in \tilde{\Delta}, \beta \in \Delta_{0}$ :

$$
\begin{aligned}
& N_{\alpha, \beta}(\zeta(u-v)-\zeta(u+v)+2 \zeta(v)) A_{k}^{\alpha+\beta}(q, u) \\
& -N_{\alpha, \beta}\left(\Phi(\alpha(q), u-v) A_{k}^{\alpha+\beta}(q, v)-\Phi(\alpha(q), u+v) A_{k}^{\alpha+\beta}(q,-v)\right) \\
& =0 .
\end{aligned}
$$

For $\alpha, \beta \in \tilde{\Delta}$ :

if $\beta=\alpha \neq-\theta \alpha$ :

$$
\begin{aligned}
& \alpha\left(K_{\alpha}\right) \Phi(\alpha(q), v) A_{k}^{\alpha}(q, u) \\
& -\frac{1}{2} N_{\theta \alpha, \alpha} \Phi(\alpha(q), u+v)\left(A_{k}^{\alpha+\theta \alpha}(q,-u)-A_{k}^{\alpha+\theta \alpha}(q, v)\right) \\
& -\sum_{j=1}^{r} \alpha_{j} A_{j}^{\alpha}(q, u) A_{k}^{\alpha}(q, v)=0 .
\end{aligned}
$$

if $\beta=-\theta \alpha$ (independently of whether $-\theta \alpha \neq \alpha$ or $-\theta \alpha=\alpha$ ):

$$
\begin{aligned}
& \frac{1}{2} \alpha_{k} \Phi^{\prime}(\alpha(q), u+v)-\alpha\left(K_{-\alpha}\right) \Phi(\alpha(q), v) A_{k}^{\alpha}(q, u) \\
& +\frac{1}{2} \Phi(\alpha(q), u+v)\left(\alpha\left(A_{k}^{\mathfrak{h}}(q, u)\right)-(\theta \alpha)\left(A_{k}^{\mathfrak{h}}(q, v)\right)\right) \\
& -\frac{1}{2} N_{\alpha,-\theta \alpha}\left(\Phi(-\alpha(q), u-v) A_{k}^{\alpha-\theta \alpha}(q, u)-\Phi(\alpha(q), u-v) A_{k}^{\alpha-\theta \alpha}(q, v)\right) \\
& +\sum_{j=1}^{r} \alpha_{j} A_{j}^{\alpha}(q, u) A_{k}^{-\theta \alpha}(q, v)=0 .
\end{aligned}
$$


if $\beta=\theta \alpha \neq-\alpha$ :

$$
\begin{aligned}
& \alpha\left(K_{\alpha}\right) \Phi(-\alpha(q), v) A_{k}^{\alpha}(q, u) \\
& +\frac{1}{2} N_{\alpha, \theta \alpha} \Phi(\alpha(q), u-v)\left(A_{k}^{\alpha+\theta \alpha}(q, u)-A_{k}^{\alpha+\theta \alpha}(q, v)\right) \\
& +\sum_{j=1}^{r} \alpha_{j} A_{j}^{\alpha}(q, u) A_{k}^{\theta \alpha}(q, v)=0 .
\end{aligned}
$$

if $\beta=-\alpha$ (independently of whether $\theta \alpha \neq-\alpha$ or $\theta \alpha=-\alpha$ ):

$$
\begin{aligned}
& \frac{1}{2} \alpha_{k} \Phi^{\prime}(\alpha(q), u-v)+\alpha\left(K_{-\alpha}\right) \Phi(-\alpha(q), v) A_{k}^{\alpha}(q, u) \\
& +\frac{1}{2} \Phi(\alpha(q), u-v)\left(\alpha\left(A_{k}^{\mathfrak{h}}(q, u)\right)-\alpha\left(A_{k}^{\mathfrak{h}}(q, v)\right)\right) \\
& -\frac{1}{2} N_{\theta \alpha,-\alpha}\left(\Phi(-\alpha(q), u+v) A_{k}^{\theta \alpha-\alpha}(q,-u)-\Phi(\alpha(q), u+v) A_{k}^{\theta \alpha-\alpha}(q, v)\right) \\
& +\sum_{j=1}^{r} \alpha_{j} A_{j}^{\alpha}(q, u) A_{k}^{-\alpha}(q, v)=0 .
\end{aligned}
$$

if $\beta \neq \pm \alpha$ and $\beta \neq \pm \theta \alpha$ :

$$
\begin{aligned}
& \alpha\left(K_{\beta}\right) \Phi(\beta(q), v) A_{k}^{\alpha}(q, u) \\
& -\frac{1}{2} N_{\alpha, \beta}\left(\Phi(\beta(q), v-u) A_{k}^{\alpha+\beta}(q, u)+\Phi(\alpha(q), u-v) A_{k}^{\alpha+\beta}(q, v)\right) \\
& -\frac{1}{2} N_{\theta \alpha, \beta}\left(\Phi(\beta(q), u+v) A_{k}^{\theta \alpha+\beta}(q,-u)-\Phi(\alpha(q), u+v) A_{k}^{\theta \alpha+\beta}(q, v)\right) \\
& -\sum_{j=1}^{r} \beta_{j} A_{j}^{\alpha}(q, u) A_{k}^{\beta}(q, v)=0 .
\end{aligned}
$$

This is a complicated set of equations which we shall solve in a series of steps.

We begin by considering the algebro-differential equations (99)-(102) for the root part of the gauge potential, which we claim to have the simple solution

$$
A_{k}^{\alpha}(q, u)=\left\{\begin{array}{cc}
\Phi(\alpha(q), u) M_{k}^{\alpha} & \text { for } \alpha \in \tilde{\Delta} \\
M_{k}^{\alpha} & \text { for } \alpha \in \Delta_{0}
\end{array}\right\}
$$

where the coefficients $M_{k}^{\alpha}$ are constants that must be determined from the remaining equations, subject to the constraint

$$
M_{k}^{\theta \alpha}=-M_{k}^{\alpha} \quad \text { for } \alpha \in \tilde{\Delta}
$$

imposed in order to guarantee the validity of eqn (91). (The corresponding constraint for $\alpha \in \Delta_{0}$ is empty.) Indeed, the statement of eqn (111) for $\alpha \in \Delta_{0}$ follows directly from 
eqns (100) and (102). Similarly, the statement of eqn (111) for $\alpha \in \tilde{\Delta}$ is an immediate consequence of eqn (99) in the degenerate case but is somewhat harder to prove in the elliptic case. To this end, we recast the functional equation (29) into the form

$$
\Phi(\alpha(q), u \mp v) \Phi(\alpha(q), \pm v)=(\zeta(u \mp v) \pm \zeta(v)) \Phi(\alpha(q), u)-\Phi^{\prime}(\alpha(q), u)
$$

and use the Ansatz

$$
A_{k}^{\alpha}(q, u)=\Phi(\alpha(q), u) M_{k}^{\alpha}(q, u) \quad \text { for } \alpha \in \tilde{\Delta}
$$

to rewrite the differential equation (99) in the form

$$
\begin{aligned}
& \Phi(\alpha(q), u) \partial_{j} M_{k}^{\alpha}(q, u)+\alpha_{j} \Phi^{\prime}(\alpha(q), u) M_{k}^{\alpha}(q, u) \\
& \quad-\frac{1}{2} \alpha_{j}(\zeta(u-v)+\zeta(u+v)) \Phi(\alpha(q), u) M_{k}^{\alpha}(q, u) \\
& \quad+\frac{1}{2} \alpha_{j}\left((\zeta(u-v)+\zeta(v)) \Phi(\alpha(q), u)-\Phi^{\prime}(\alpha(q), u)\right) M_{k}^{\alpha}(q, v) \\
& \quad+\frac{1}{2} \alpha_{j}\left((\zeta(u+v)-\zeta(v)) \Phi(\alpha(q), u)-\Phi^{\prime}(\alpha(q), u)\right) M_{k}^{\alpha}(q,-v) \\
& \quad 0
\end{aligned}
$$

while the algebraic equation (101) takes the form

$$
\begin{aligned}
& (\zeta(u-v)-\zeta(u+v)+2 \zeta(v)) \Phi(\alpha(q), u) M_{k}^{\alpha}(q, u) \\
& \quad-\left((\zeta(u-v)+\zeta(v)) \Phi(\alpha(q), u)-\Phi^{\prime}(\alpha(q), u)\right) M_{k}^{\alpha}(q, v) \\
& \quad+\left((\zeta(u+v)-\zeta(v)) \Phi(\alpha(q), u)-\Phi^{\prime}(\alpha(q), u)\right) M_{k}^{\alpha}(q,-v) \\
& \quad+0 .
\end{aligned}
$$

Both of these equations can be simplified by using the functional equation (30) and subtracting $\frac{1}{2} \alpha_{j}$ times the second from the first, with the result that

$$
\begin{aligned}
\partial_{j} M_{k}^{\alpha}(q, u)= & \alpha_{j}(\zeta(u-v)+\zeta(v)+\zeta(\alpha(q))-\zeta(\alpha(q)+u)) \\
& \times\left(M_{k}^{\alpha}(q, u)-M_{k}^{\alpha}(q, v)\right)
\end{aligned}
$$

while

$$
\begin{aligned}
& (\zeta(u-v)-\zeta(u+v)+2 \zeta(v)) M_{k}^{\alpha}(q, u) \\
& \quad-(\zeta(u-v)+\zeta(v)-\zeta(\alpha(q)+u)+\zeta(\alpha(q))) M_{k}^{\alpha}(q, v) \\
& \quad+(\zeta(u+v)-\zeta(v)-\zeta(\alpha(q)+u)+\zeta(\alpha(q))) M_{k}^{\alpha}(q,-v) \\
& \quad=0
\end{aligned}
$$


Antisymmetrizing the last equation with respect to the exchange of $u$ and $-u$ gives

$$
\begin{aligned}
(\zeta(u-v)-\zeta(u+v)+2 \zeta(v))\left(M_{k}^{\alpha}(q, u)-M_{k}^{\alpha}(q,-u)\right) \\
=(\zeta(u-v)+\zeta(u+v)+\zeta(\alpha(q)-u)-\zeta(\alpha(q)+u)) \\
\quad \times\left(M_{k}^{\alpha}(q, v)-M_{k}^{\alpha}(q,-v)\right) .
\end{aligned}
$$

In order to analyze the consequences of this relation, we shall use the identity

$$
\zeta(x+y)-\zeta(x)-\zeta(y)=\frac{1}{2} \frac{\wp^{\prime}(x)-\wp^{\prime}(y)}{\wp(x)-\wp(y)},
$$

which can also be written as

$$
\zeta(u-v)-\zeta(u+v)+2 \zeta(v)=\frac{\wp^{\prime}(v)}{\wp(u)-\wp(v)},
$$

implying that

$$
\begin{aligned}
& \zeta(u-v)+\zeta(u+v)+\zeta(s-u)-\zeta(s+u) \\
& \quad=\frac{\wp^{\prime}(u)}{\wp(u)-\wp(v)}+\frac{\wp^{\prime}(u)}{\wp(s)-\wp(u)}=\frac{\wp^{\prime}(u)(\wp(s)-\wp(v))}{(\wp(s)-\wp(u))(\wp(u)-\wp(v))} .
\end{aligned}
$$

After rearranging coefficients, we conclude that eqn (115) can be reformulated as stating that the function

$$
\frac{\wp(\alpha(q))-\wp(u)}{\wp^{\prime}(u)}\left(M_{k}^{\alpha}(q, u)-M_{k}^{\alpha}(q,-u)\right)
$$

is independent of $u$. Putting $u=\alpha(q)$, we see that it must actually vanish identically, which is only possible if

$$
M_{k}^{\alpha}(q, u)-M_{k}^{\alpha}(q,-u)=0
$$

Inserting this result back into eqn (114), we get

$$
M_{k}^{\alpha}(q, u)-M_{k}^{\alpha}(q, v)=0
$$

Now eqn (113) implies that $M_{k}^{\alpha}$ is in fact a constant.

For what follows, we shall find it convenient to assemble the constants introduced in eqn (111) above into a vector in $\mathfrak{a}_{0}$ by writing, for any $\alpha \in \Delta$,

$$
M_{\alpha}=\sum_{j=1}^{r} M_{j}^{\alpha} H_{j}
$$

so that of course

$$
M_{k}^{\alpha}=\left(H_{k}, M_{\alpha}\right) .
$$


In analogy with eqn (88), we also introduce the abbreviation

$$
M_{\alpha}^{ \pm}=\frac{1}{2}\left(M_{\alpha} \pm M_{-\alpha}\right) .
$$

Note that, for $\alpha \in \tilde{\Delta}$, the generators $M_{\alpha} \in \mathfrak{a}_{0}$ are in a sense complementary to the generators $K_{\alpha} \in \mathrm{ib}_{0}$. (This observation will come to play an important role later on.) In particular, eqn (112) amounts to the condition

$$
M_{\theta \alpha}=-M_{\alpha} \quad \text { for } \alpha \in \tilde{\Delta}
$$

which is analogous to the condition

$$
K_{\theta \alpha}=K_{\alpha} \quad \text { for } \alpha \in \tilde{\Delta}
$$

of Ref. [1].

Now we are ready to state the first main result of this section. Our terminology will follow that of Ref. [8], according to which roots $\alpha \in \Delta$ are called imaginary if $\theta \alpha=\alpha$, real if $\theta \alpha=-\alpha$ and complex if $\theta \alpha$ and $\alpha$ are linearly independent, whereas two roots $\alpha$ and $\beta$ are called strongly orthogonal if both $\alpha+\beta$ and $\alpha-\beta$ are not roots (as is well known, this implies that $\alpha$ and $\beta$ are orthogonal in the usual sense).

Proposition 2 The integrable Calogero model associated with the root system of a symmetric pair $(\mathfrak{g}, \theta)$ admits a gauge transformation $g$ from the standard Lax pair of Olshanetsky and Perelomov and the dynamical R-matrix of Ref. [1] to a new Lax pair with a numerical R-matrix if and only if a) the automorphism $\theta$ acts on the root system $\Delta$ in such a way that

- $\Delta$ contains no imaginary roots, i.e., $\Delta_{0}=\emptyset$ and $\tilde{\Delta}=\Delta$,

- for any complex root $\alpha$ in $\Delta, \theta \alpha$ and $\alpha$ are strongly orthogonal, i.e., $\theta \alpha \pm \alpha \notin \Delta$,

and b) the set of generators $K_{\alpha} \in \mathrm{ib}_{0}$ appearing in eqns (85) and (87) can be complemented by a set of generators $M_{\alpha} \in \mathfrak{a}_{0}$ which, taken together, satisfy the algebraic constraints

$$
\begin{gathered}
\alpha\left(K_{\alpha}^{+}\right)=0, \quad \alpha\left(M_{\alpha}^{+}\right)=0, \\
K_{\alpha}^{-}=\frac{\epsilon_{\alpha}}{\sqrt{2}|\alpha|}\left(H_{\alpha}\right)_{\mathfrak{b}}, \quad M_{\alpha}^{-}=\frac{\epsilon_{\alpha}}{\sqrt{2}|\alpha|}\left(H_{\alpha}\right)_{\mathfrak{a}}, \\
\alpha\left(K_{\beta}\right) K_{\alpha}-\beta\left(K_{\alpha}\right) K_{\beta}=\frac{1}{2}\left(N_{\alpha, \beta} K_{\alpha+\beta}+N_{\theta \alpha, \beta} K_{\theta \alpha+\beta}\right) \\
\alpha\left(K_{\beta}\right) M_{\alpha}-\beta\left(M_{\alpha}\right) M_{\beta}=\frac{1}{2}\left(N_{\alpha, \beta} M_{\alpha+\beta}-N_{\theta \alpha, \beta} M_{\theta \alpha+\beta}\right) \\
\text { for } \alpha, \beta \in \Delta \text { such that } \beta \neq \pm \alpha, \beta \neq \pm \theta \alpha,
\end{gathered}
$$


as well as the additional algebraic constraints

$$
\begin{gathered}
\sum_{\alpha \in \Delta}\left(H_{\alpha}\right)_{\mathfrak{a}} \otimes K_{\alpha} \otimes M_{-\alpha}=0, \sum_{\alpha \in \Delta}\left(H_{\alpha}\right)_{\mathfrak{b}} \otimes M_{\alpha} \otimes M_{-\alpha}=0, \\
\sum_{\substack{\beta, \gamma \in \Delta \\
\beta+\gamma=\alpha}} N_{\beta, \gamma} M_{\beta} \otimes K_{\gamma}=\left(H_{\alpha}\right)_{\mathfrak{a}} \otimes K_{\alpha}-M_{\alpha} \otimes\left(H_{\alpha}\right)_{\mathfrak{b}}, \\
\sum_{\substack{\beta, \gamma \in \Delta \\
\beta+\gamma=\alpha}} N_{\beta, \gamma} M_{\beta} \otimes M_{\gamma}=\left(H_{\alpha}\right)_{\mathfrak{a}} \otimes M_{\alpha}-M_{\alpha} \otimes\left(H_{\alpha}\right)_{\mathfrak{a}},
\end{gathered}
$$

to be imposed in the case of the elliptic model, where $K_{\alpha}^{ \pm}=\frac{1}{2}\left(K_{\alpha} \pm K_{-\alpha}\right)$ and $M_{\alpha}^{ \pm}=\frac{1}{2}\left(M_{\alpha} \pm M_{-\alpha}\right)$ as above, with $\epsilon_{\alpha}= \pm 1$. In this case, the root part and the Cartan part of the potential $A_{k}=g^{-1} \partial_{k} g$ associated with this gauge transformation $g$ are given by

$$
A_{k}^{\alpha}(q)=w(\alpha(q))\left(H_{k}, M_{\alpha}\right)
$$

and

$$
A_{k}^{\mathfrak{h}}(q)=\sum_{\alpha \in \Delta} \frac{w^{\prime}(\alpha(q))}{w(\alpha(q))}\left(H_{k}, M_{-\alpha}\right) K_{\alpha}
$$

for the degenerate model and by

$$
A_{k}^{\alpha}(q, u)=\Phi(\alpha(q), u)\left(H_{k}, M_{\alpha}\right)
$$

and

$$
A_{k}^{\mathfrak{h}}(q, u)=-\sum_{\alpha \in \Delta} \zeta(\alpha(q))\left(H_{k}, M_{-\alpha}\right) K_{\alpha}-\zeta(u) H_{k}
$$

for the elliptic model.

Note. As we shall show after completing the proof of Proposition 2, eqn (123) forces all roots $\alpha$ in $\Delta$ to have the same length (which by convention we fix to be $\sqrt{2}$ ) and also allows for a choice of basis in which the signs $\epsilon_{\alpha}$ are independent of $\alpha$. so that eqn (122) can be simplified as follows:

$$
K_{\alpha}^{-}=\frac{1}{2} \epsilon\left(H_{\alpha}\right)_{\mathfrak{b}}, \quad M_{\alpha}^{-}=\frac{1}{2} \epsilon\left(H_{\alpha}\right)_{\mathfrak{a}} .
$$

Proof. With the vector notation introduced above, we can first of all use eqn (102), with $\alpha$ replaced by $\alpha+\theta \alpha$, to conclude that the middle terms in eqns (106) and (108) vanish identically and thus reduce both of them to a single algebraic constraint:

$$
\alpha\left(K_{\alpha}\right)=\alpha\left(M_{\alpha}\right) \quad \text { for } \alpha \in \tilde{\Delta} \text { such that } \theta \alpha \neq-\alpha .
$$

Note that replacing $\alpha$ by $-\alpha$ and adding/subtracting the two equations, we get

$$
\begin{array}{ll}
\alpha\left(K_{\alpha}^{+}\right)=\alpha\left(M_{\alpha}^{+}\right) & \text {for } \alpha \in \tilde{\Delta} \text { such that } \theta \alpha \neq-\alpha, \\
\alpha\left(K_{\alpha}^{-}\right)=\alpha\left(M_{\alpha}^{-}\right) & \text {for } \alpha \in \tilde{\Delta} \text { such that } \theta \alpha \neq-\alpha .
\end{array}
$$


Using eqn (89), the first of these can be sharpened as follows:

$$
\alpha\left(K_{\alpha}^{+}\right)=0=\alpha\left(M_{\alpha}^{+}\right) \quad \text { for } \alpha \in \tilde{\Delta} \text { such that } \theta \alpha-\alpha \notin \Delta .
$$

(Indeed, if $\theta \alpha=-\alpha$ so that eqn (131) no longer applies, eqn (134) remains correct because in this case $M_{\alpha}^{+}=0$, according to eqns (118) and (1119).) Next, inserting eqn (111) together with the functional equation (25) into the differential equation (95) for the Cartan part of the gauge potential, we see that this equation can be solved by setting

$$
A_{k}^{\mathfrak{h}}(q, u)=-\sum_{\alpha \in \tilde{\Delta}} \zeta(\alpha(q)) M_{k}^{-\alpha} K_{\alpha}-M_{k}^{\mathfrak{h}}(u),
$$

where the $M_{k}^{\mathfrak{h}}(u)$ are constants that must be determined from the remaining equations, provided we assume the coefficients $M_{k}^{\alpha}$ to satisfy the relation

$$
\sum_{\alpha \in \tilde{\Delta}} \alpha_{j} M_{k}^{-\alpha} K_{\alpha}=0 \quad \text { for } 1 \leqslant j, k \leqslant r .
$$

Converted into a tensor equation, it reads

$$
\sum_{\alpha \in \tilde{\Delta}}\left(H_{\alpha}\right)_{\mathfrak{a}} \otimes K_{\alpha} \otimes M_{-\alpha}=0,
$$

which leads back to eqn (136) by taking the scalar product with $H_{j}$ in the first and with $H_{k}$ in the third tensor factor. Note that in the degenerate case, the same argument works, but eqn (136/137) is not needed. Moreover, eqn (96) is satisfied as a consequence of the identity

$$
\sum_{\alpha \in \tilde{\Delta}} \alpha_{j} f(\alpha(q)) M_{k}^{-\alpha} K_{\alpha}=0 \quad \text { for } r+1 \leqslant j \leqslant r+s,
$$

which is valid for any even function $f$ (such as $\zeta^{\prime}$ ): this is easily shown by replacing $\alpha$ by $\theta \alpha$ in the sum and noting that $(\theta \alpha)_{j}=\alpha_{j}$ for $r+1 \leqslant j \leqslant r+s,(\theta \alpha)(q)=-\alpha(q)$, $M_{k}^{-\theta \alpha}=-M_{k}^{-\alpha}$ and $K_{\theta \alpha}=K_{\alpha}$, so that

$$
\sum_{\alpha \in \tilde{\Delta}} \alpha_{j} f(\alpha(q)) M_{k}^{-\alpha} K_{\alpha}=-\sum_{\alpha \in \tilde{\Delta}} \alpha_{j} f(\alpha(q)) M_{k}^{-\alpha} K_{\alpha} \quad \text { for } r+1 \leqslant j \leqslant r+s .
$$

Similarly, inserting eqn (111) together with the functional equation (29) into eqns (107) and (109), we obtain

$$
\begin{aligned}
& \frac{1}{2} \alpha_{k}(-\Phi(\alpha(q), u) \Phi(\alpha(q), v)+(\zeta(u)+\zeta(v)) \Phi(\alpha(q), u+v)) \\
& -\alpha\left(K_{-\alpha}\right) M_{k}^{\alpha} \Phi(\alpha(q), u) \Phi(\alpha(q), v) \\
& -\frac{1}{2} \Phi(\alpha(q), u+v)\left(\alpha\left(M_{k}^{\mathfrak{h}}(u)\right)-(\theta \alpha)\left(M_{k}^{\mathfrak{h}}(v)\right)\right) \\
& +\frac{1}{2} N_{\alpha,-\theta \alpha} M_{k}^{\alpha-\theta \alpha}(-\Phi(-\alpha(q), u-v) \Phi(2 \alpha(q), u)-\Phi(-\alpha(q), v-u) \Phi(2 \alpha(q), v)) \\
& -\alpha\left(M_{\alpha}\right) M_{k}^{-\alpha} \Phi(\alpha(q), u) \Phi(\alpha(q), v)=0
\end{aligned}
$$


and

$$
\begin{aligned}
& \frac{1}{2} \alpha_{k}(-\Phi(\alpha(q), u) \Phi(\alpha(q),-v)+(\zeta(u)-\zeta(v)) \Phi(\alpha(q), u-v)) \\
& -\alpha\left(K_{-\alpha}\right) M_{k}^{\alpha} \Phi(\alpha(q), u) \Phi(\alpha(q),-v) \\
& -\frac{1}{2} \Phi(\alpha(q), u-v)\left(\alpha\left(a_{k}^{\mathfrak{h}}(u)\right)-\alpha\left(a_{k}^{\mathfrak{h}}(v)\right)\right) \\
& -\frac{1}{2} N_{\theta \alpha,-\alpha} M_{k}^{\theta \alpha-\alpha}(-\Phi(-\alpha(q), u+v) \Phi(2 \alpha(q), u)-\Phi(-\alpha(q),-u-v) \Phi(2 \alpha(q),-v)) \\
& -\alpha\left(M_{\alpha}\right) M_{k}^{-\alpha} \Phi(\alpha(q), u) \Phi(\alpha(q),-v)=0
\end{aligned}
$$

respectively. In both cases, the terms proportional to $\Phi(\alpha(q), u \pm v)$ cancel provided we set

$$
M_{k}^{\mathfrak{h}}(u)=\zeta(u) H_{k}
$$

which also guarantees that eqn (103) is valid, and due to the functional equation (28), the remaining terms then cancel if we impose the relation

$$
\frac{1}{2} \alpha_{k}+\alpha\left(K_{-\alpha}\right) M_{k}^{\alpha}+\alpha\left(M_{\alpha}\right) M_{k}^{-\alpha}+\frac{1}{2} N_{\theta \alpha,-\alpha} M_{k}^{\theta \alpha-\alpha}=0 \quad \text { for } \alpha \in \tilde{\Delta} .
$$

Converted to a vector equation in $\mathfrak{a}_{0}$, it reads

$$
\frac{1}{2}\left(H_{\alpha}\right)_{\mathfrak{a}}+\alpha\left(K_{-\alpha}\right) M_{\alpha}+\alpha\left(M_{\alpha}\right) M_{-\alpha}+\frac{1}{2} N_{\theta \alpha,-\alpha} M_{\theta \alpha-\alpha}=0 \quad \text { for } \alpha \in \tilde{\Delta},
$$

which leads back to eqn (139) by taking the scalar product with $H_{k}$. Even simpler to handle is eqn (110), which by insertion of eqn (111) together with the functional equation (28) reduces to the relation

$$
\begin{gathered}
\alpha\left(K_{\beta}\right) M_{k}^{\alpha}-\frac{1}{2}\left(N_{\alpha, \beta} M_{k}^{\alpha+\beta}-N_{\theta \alpha, \beta} M_{k}^{\theta \alpha+\beta}\right)-\beta\left(M_{\alpha}\right) M_{k}^{\beta}=0 \\
\text { for } \alpha, \beta \in \tilde{\Delta} \text { such that } \beta \neq \pm \alpha, \beta \neq \pm \theta \alpha .
\end{gathered}
$$

Converted to a vector equation in $\mathfrak{a}_{0}$, it reads

$$
\begin{gathered}
\alpha\left(K_{\beta}\right) M_{\alpha}-\frac{1}{2}\left(N_{\alpha, \beta} M_{\alpha+\beta}-N_{\theta \alpha, \beta} M_{\theta \alpha+\beta}\right)-\beta\left(M_{\alpha}\right) M_{\beta}=0 \\
\text { for } \alpha, \beta \in \tilde{\Delta} \text { such that } \beta \neq \pm \alpha, \beta \neq \pm \theta \alpha
\end{gathered}
$$

which leads back to eqn (141) by taking the scalar product with $H_{k}$. Finally, inserting eqn (111) together with the functional equation (29) (more precisely, the difference of two copies of eqn (29): one with $v \rightarrow u, u \rightarrow v$ and one with $v \rightarrow u, u \rightarrow-v$ ) into eqn (105) and dividing by $\Phi(\alpha(q), u)$, we obtain

$$
N_{\alpha, \beta} M_{k}^{\alpha+\beta}=0 \quad \text { for } \alpha \in \tilde{\Delta}, \beta \in \Delta_{0},
$$

which in turn reduces eqn (104) to

$$
\alpha\left(K_{\beta}\right) M_{k}^{\alpha}=\beta\left(M_{\alpha}\right) M_{k}^{\beta} \quad \text { for } \alpha \in \Delta_{0}, \beta \in \tilde{\Delta} .
$$


In the degenerate case, the same conclusion is reached along a slightly different path, since in this case eqn (105) is void while eqn (104) takes the form

$$
\alpha\left(K_{\beta}\right) w(\beta(q)) M_{k}^{\alpha}+N_{\alpha, \beta} M_{k}^{\alpha+\beta} w^{\prime}(\beta(q))-\beta\left(M_{\alpha}\right) w(\beta(q))=0
$$

which again leads to eqns (143) and (144) since $w$ and $w^{\prime}$ are functionally independent.

Before proceeding to the solution of the remaining equations, let us pause to draw a few consequences of the algebraic constraints (131)-(134), (139/140) and (143) derived so far; this will help us considerably to simplify our further work. For this purpose, we must distinguish between real and complex roots $\alpha$ in $\tilde{\Delta}$ :

- For real roots $\alpha \in \Delta(\theta \alpha=-\alpha)$, eqns (119) and (120), together with the fact that $K_{\alpha} \in \mathrm{ib} \mathfrak{b}_{0}$ and hence $\theta K_{\alpha}=K_{\alpha}$, imply that $M_{-\alpha}=-M_{\alpha}, K_{-\alpha}=K_{\alpha}$ and $\alpha\left(K_{\alpha}\right)=\alpha\left(\theta K_{\alpha}\right)=\theta \alpha\left(K_{\alpha}\right)=-\alpha\left(K_{\alpha}\right)$, so $\alpha\left(K_{ \pm \alpha}\right)=0$. Thus in this case, the last term and the second term in eqn (140) drop out, so we get

$$
\alpha\left(M_{\alpha}\right) M_{\alpha}=\frac{1}{2}\left(H_{\alpha}\right)_{\mathfrak{a}} .
$$

Applying $\alpha$ to this relation and using that in this case, $\left(H_{\alpha}\right)_{\mathfrak{a}}=H_{\alpha}$, we conclude that

$$
2 \alpha\left(M_{\alpha}\right)^{2}=\alpha\left(H_{\alpha}\right)=(\alpha, \alpha)
$$

i.e.,

$$
\alpha\left(M_{\alpha}\right)=\frac{\epsilon_{\alpha}}{\sqrt{2}}|\alpha| \quad \text { and } \quad M_{\alpha}=\frac{\epsilon_{\alpha}}{\sqrt{2}|\alpha|}\left(H_{\alpha}\right)_{\mathfrak{a}}
$$

where $\epsilon_{\alpha}=\epsilon_{-\alpha}=\epsilon_{\theta \alpha}=\epsilon_{-\theta \alpha}$ is a sign factor $( \pm 1)$.

- For complex roots $\alpha \in \Delta(\theta \alpha \neq \pm \alpha)$, eqn (131) implies that $\alpha\left(K_{-\alpha}\right)=\alpha\left(M_{-\alpha}\right)$, so we may rewrite eqn (140) in the form

$$
\frac{1}{2}\left(H_{\alpha}\right)_{\mathfrak{a}}+2 \alpha\left(M_{\alpha}^{+}\right) M_{\alpha}^{+}-2 \alpha\left(M_{\alpha}^{-}\right) M_{\alpha}^{-}+\frac{1}{2} N_{\theta \alpha,-\alpha} M_{\theta \alpha-\alpha}=0 .
$$

But under the substitution $\alpha \rightarrow-\alpha$, the first three terms in this equation are odd while the last is even, which forces them to vanish separately. Now we must distinguish two cases:

- If $\theta \alpha-\alpha$ is not a root, the last term drops out and, according to eqn (134), so does the second. Thus we get

$$
\alpha\left(M_{\alpha}^{-}\right) M_{\alpha}^{-}=\frac{1}{4}\left(H_{\alpha}\right)_{\mathfrak{a}}
$$

Applying $\alpha$ to this relation, we conclude that

$$
4 \alpha\left(M_{\alpha}^{-}\right)^{2}=\alpha\left(\left(H_{\alpha}\right)_{\mathfrak{a}}\right)=\frac{1}{2}(\alpha, \alpha-\theta \alpha)
$$


which can be further simplified because $\theta \alpha+\alpha$ is never a root 9, Ex. F.2, p. 530] so that in this case, $\theta \alpha$ and $\alpha$ are (strongly) orthogonal, leading to

$$
\alpha\left(M_{\alpha}^{-}\right)=\frac{\epsilon_{\alpha}}{2 \sqrt{2}}|\alpha| \quad \text { and } \quad M_{\alpha}^{-}=\frac{\epsilon_{\alpha}}{\sqrt{2}|\alpha|}\left(H_{\alpha}\right)_{\mathfrak{a}},
$$

where $\epsilon_{\alpha}=\epsilon_{-\alpha}=\epsilon_{\theta \alpha}=\epsilon_{-\theta \alpha}$ is a sign factor $( \pm 1)$.

- If $\theta \alpha-\alpha$ is a root, we arrive at a contradiction, since in this case $\theta \alpha-\alpha$ is a real root, so that according to the previous item, $M_{\theta \alpha-\alpha}$ cannot vanish. Therefore, this possibility must be excluded.

Moreover, we see that $M_{\alpha}$ can never vanish, so the only way to guarantee the validity of eqn (143) is to assume that the sum of a root $\alpha$ in $\tilde{\Delta}$ and a $\operatorname{root} \beta$ in $\Delta_{0}$ is never a root. Since we may freely change the sign of $\beta$, this forces all roots in $\tilde{\Delta}$ to be (strongly) orthogonal to all roots in $\Delta_{0}$, which is only possible if one of these two sets is empty, since $\mathfrak{g}$ is supposed to be simple and hence $\Delta$ must be irreducible. This proves the two restrictions on the action of $\theta$ on $\Delta$ stated in the proposition, namely

$$
\Delta_{0}=\emptyset, \tilde{\Delta}=\Delta
$$

and, for any root $\alpha$ in $\Delta$,

$$
\theta \alpha \pm \alpha \notin \Delta \quad \text { and either } \theta \alpha=-\alpha \text { or } \theta \alpha \perp \alpha
$$

Moroever, eqns (145) and (146) can be unified into a single formula

$$
M_{\alpha}^{-}=\frac{\epsilon_{\alpha}}{\sqrt{2}|\alpha|}\left(H_{\alpha}\right)_{\mathfrak{a}}
$$

where $\epsilon_{\alpha}=\epsilon_{-\alpha}=\epsilon_{\theta \alpha}=\epsilon_{-\theta \alpha}$ is a sign factor $( \pm 1)$.

Let us summarize the results obtained so far. With the exception of eqn (97), the system of equations (95)-(110) has been completely solved in terms of the algebraic conditions (147), (148), the explicit formulae (126)-(129) for the gauge potential with the explicit formula (149) for the odd part $M_{\alpha}^{-}$of the coefficient vectors $M_{\alpha}$ and the algebraic constraints (121), (133), (136/137) and (141/142). Thus we are left with the task of verifying the implications of eqns (14), (15) and (97). 
Beginning with eqn (14), we use the functional equation (25) and eqns (147)-(149) to compute

$$
\begin{aligned}
& \partial_{k} A_{l}^{\mathfrak{h}}(q, u)-\partial_{l} A_{k}^{\mathfrak{h}}(q, u)+\sum_{\alpha \in \Delta} A_{k}^{\alpha}(q, u) A_{l}^{-\alpha}(q, u) H_{\alpha} \\
& =\sum_{\alpha \in \Delta} \zeta^{\prime}(\alpha(q))\left(\alpha_{l}\left(H_{k}, M_{-\alpha}\right) K_{\alpha}-\alpha_{k}\left(H_{l}, M_{-\alpha}\right) K_{\alpha}\right) \\
& +\sum_{\alpha \in \Delta} \Phi(\alpha(q), u) \Phi(-\alpha(q), u)\left(H_{k}, M_{\alpha}\right)\left(H_{l}, M_{-\alpha}\right) H_{\alpha} \\
& =\frac{1}{2} \sum_{\alpha \in \Delta} \zeta^{\prime}(\alpha(q))\left(+\alpha_{l}\left(H_{k}, M_{-\alpha}\right) K_{\alpha}-\alpha_{l}\left(H_{k}, M_{\alpha}\right) K_{-\alpha}\right. \\
& \left.+\left(H_{k}, M_{\alpha}\right)\left(H_{l}, M_{-\alpha}\right) H_{\alpha}-\left(H_{k}, M_{-\alpha}\right)\left(H_{l}, M_{\alpha}\right) H_{\alpha}\right) \\
& -\zeta^{\prime}(u) \sum_{\alpha \in \Delta}\left(H_{k}, M_{\alpha}\right)\left(H_{l}, M_{-\alpha}\right) H_{\alpha} \\
& =\sum_{\alpha \in \Delta} \zeta^{\prime}(\alpha(q))\left(-\alpha_{l}\left(H_{k}, M_{\alpha}^{-}\right) K_{\alpha}^{+}+\alpha_{l}\left(H_{k}, M_{\alpha}^{+}\right) K_{\alpha}^{-}\right. \\
& +\alpha_{k}\left(H_{l}, M_{\alpha}^{-}\right) K_{\alpha}^{+}-\alpha_{k}\left(H_{l}, M_{\alpha}^{+}\right) K_{\alpha}^{-} \\
& \left.+\left(H_{k}, M_{\alpha}^{-}\right)\left(H_{l}, M_{\alpha}^{+}\right) H_{\alpha}-\left(H_{k}, M_{\alpha}^{+}\right)\left(H_{l}, M_{\alpha}^{-}\right) H_{\alpha}\right) \\
& -\zeta^{\prime}(u) \sum_{\alpha \in \Delta}\left(H_{k}, M_{\alpha}\right)\left(H_{l}, M_{-\alpha}\right)\left(H_{\alpha}\right)_{\mathfrak{b}} \\
& =\sum_{\alpha \in \Delta} \zeta^{\prime}(\alpha(q))\left(-\frac{\epsilon_{\alpha}}{\sqrt{2}|\alpha|} \alpha_{l} \alpha_{k} K_{\alpha}^{+}+\frac{\epsilon_{\alpha}}{\sqrt{2}|\alpha|} \alpha_{k} \alpha_{l} K_{\alpha}^{+}\right) \\
& +\sum_{\alpha \in \Delta} \zeta^{\prime}(\alpha(q))\left(+\alpha_{l}\left(H_{k}, M_{\alpha}^{+}\right) K_{\alpha}^{-}-\alpha_{k}\left(H_{l}, M_{\alpha}^{+}\right) K_{\alpha}^{-}\right. \\
& \left.+\frac{\epsilon_{\alpha}}{\sqrt{2}|\alpha|} \alpha_{k}\left(H_{l}, M_{\alpha}^{+}\right)\left(H_{\alpha}\right)_{\mathfrak{b}}-\frac{\epsilon_{\alpha}}{\sqrt{2}|\alpha|} \alpha_{l}\left(H_{k}, M_{\alpha}^{+}\right)\left(H_{\alpha}\right)_{\mathfrak{b}}\right) \\
& -\zeta^{\prime}(u) \sum_{\alpha \in \Delta}\left(H_{k}, M_{\alpha}\right)\left(H_{l}, M_{-\alpha}\right)\left(H_{\alpha}\right)_{\mathfrak{b}} .
\end{aligned}
$$

Obviously, the whole expression will vanish provided we assume that

$$
K_{\alpha}^{-}=\frac{\epsilon_{\alpha}}{\sqrt{2}|\alpha|}\left(H_{\alpha}\right)_{\mathfrak{b}}
$$

which is complementary to the condition (149) derived previously and that

$$
\sum_{\alpha \in \Delta}\left(H_{\alpha}\right)_{\mathfrak{b}} \otimes M_{\alpha} \otimes M_{-\alpha}=0
$$


which is complementary to the condition (137) derived previously. Note that in the degenerate case, the same argument works, but eqn (151) is not needed. Note also that eqn (133) can now be eliminated because it follows from eqns (148), (149) and (150).

For the proof of eqn (15), the trick is to split the sum over roots $\beta$ coming from the third and fourth term into various pieces: the contribution with $\beta=\alpha$ and, if $\theta \alpha \neq-\alpha$, also the contribution with $\beta=\theta \alpha$, which cancel mutually, the contribution with $\beta=-\alpha$ and, if $\theta \alpha \neq-\alpha$, also the contribution with $\beta=-\theta \alpha$, which combine with the contributions coming from the first and second term (transformed using the functional equation (30)), and finally the remaining contributions with $\beta \neq \pm \alpha$ and $\beta \neq \pm \theta \alpha$ : these can be complemented by terms that also cancel mutually (marked by underlining) and then be combined with the contributions from the fifth term (transformed using the functional equation (31)):

$$
\begin{aligned}
& \partial_{k} A_{l}^{\alpha}(q, u)-\partial_{l} A_{k}^{\alpha}(q, u)+\alpha\left(A_{k}^{\mathfrak{h}}(q, u)\right) A_{l}^{\alpha}(q, u)-\alpha\left(A_{l}^{\mathfrak{h}}(q, u)\right) A_{k}^{\alpha}(q, u) \\
& +\sum_{\substack{\beta, \gamma \in \Delta \\
\beta+\gamma=\alpha}} N_{\beta, \gamma} A_{k}^{\beta}(q, u) A_{l}^{\gamma}(q, u) \\
& =\Phi^{\prime}(\alpha(q), u) \alpha_{k}\left(H_{l}, M_{\alpha}\right)-\Phi^{\prime}(\alpha(q), u) \alpha_{l}\left(H_{k}, M_{\alpha}\right) \\
& +\Phi(\alpha(q), u)\left(\sum_{\beta \in \Delta} \zeta(\beta(q)) \alpha\left(K_{-\beta}\right)\left(H_{k}, M_{\beta}\right)\left(H_{l}, M_{\alpha}\right)-\zeta(u) \alpha_{k}\left(H_{l}, M_{\alpha}\right)\right) \\
& -\Phi(\alpha(q), u)\left(\sum_{\beta \in \Delta} \zeta(\beta(q)) \alpha\left(K_{-\beta}\right)\left(H_{l}, M_{\beta}\right)\left(H_{k}, M_{\alpha}\right)-\zeta(u) \alpha_{l}\left(H_{k}, M_{\alpha}\right)\right) \\
& +\sum_{\substack{\gamma, \delta \in \Delta \\
\gamma+\delta=\alpha}} N_{\gamma, \delta} \Phi(\gamma(q), u) \Phi(\delta(q), u)\left(H_{k}, M_{\gamma}\right)\left(H_{l}, M_{\delta}\right) \\
& =\left(\Phi^{\prime}(\alpha(q), u)-\Phi(\alpha(q), u) \zeta(u)\right)\left(\left(H_{k}, H_{\alpha}\right)\left(H_{l}, M_{\alpha}\right)-\left(H_{l}, H_{\alpha}\right)\left(H_{k}, M_{\alpha}\right)\right) \\
& +\Phi(\alpha(q), u) \zeta(\alpha(q)) \\
& \times\left(+\alpha\left(K_{-\alpha}\right)\left(H_{k}, M_{\alpha}\right)\left(H_{l}, M_{\alpha}\right)\right. \\
& \text { - } \alpha\left(K_{\alpha}\right)\left(H_{k}, M_{-\alpha}\right)\left(H_{l}, M_{\alpha}\right) \\
& \text { - }\left(1-\delta_{\theta \alpha,-\alpha}\right) \alpha\left(K_{-\theta \alpha}\right)\left(H_{k}, M_{\theta \alpha}\right)\left(H_{l}, M_{\alpha}\right) \\
& +\left(1-\delta_{\theta \alpha,-\alpha}\right) \alpha\left(K_{\theta \alpha}\right)\left(H_{k}, M_{-\theta \alpha}\right)\left(H_{l}, M_{\alpha}\right) \\
& \text { - } \alpha\left(K_{-\alpha}\right)\left(H_{l}, M_{\alpha}\right)\left(H_{k}, M_{\alpha}\right) \\
& +\alpha\left(K_{\alpha}\right)\left(H_{l}, M_{-\alpha}\right)\left(H_{k}, M_{\alpha}\right) \\
& +\left(1-\delta_{\theta \alpha,-\alpha}\right) \alpha\left(K_{-\theta \alpha}\right)\left(H_{l}, M_{\theta \alpha}\right)\left(H_{k}, M_{\alpha}\right) \\
& \left.-\left(1-\delta_{\theta \alpha,-\alpha}\right) \alpha\left(K_{\theta \alpha}\right)\left(H_{l}, M_{-\theta \alpha}\right)\left(H_{k}, M_{\alpha}\right)\right)
\end{aligned}
$$




$$
\begin{aligned}
& +\Phi(\alpha(q), u) \sum_{\substack{\beta \in \Delta \\
\beta \neq \pm \alpha, \beta \neq \pm \theta \alpha}} \zeta(\beta(q))\left(H_{k}, M_{\beta}\right)\left(\alpha\left(K_{-\beta}\right)\left(H_{l}, M_{\alpha}\right)+\underline{\beta\left(M_{\alpha}\right)\left(H_{l}, M_{-\beta}\right)}\right) \\
& -\Phi(\alpha(q), u) \sum_{\substack{\beta \in \Delta \\
\beta \neq \pm \alpha, \beta \neq \pm \theta \alpha}} \zeta(\beta(q))\left(H_{l}, M_{\beta}\right)\left(\alpha\left(K_{-\beta}\right)\left(H_{k}, M_{\alpha}\right)+\underline{\beta\left(M_{\alpha}\right)\left(H_{k}, M_{-\beta}\right)}\right) \\
& +\Phi(\alpha(q), u) \sum_{\substack{\gamma, \delta \in \Delta \\
\gamma+\delta=\alpha}}(\zeta(\gamma(q))+\zeta(\delta(q))) N_{\gamma, \delta}\left(H_{k}, M_{\gamma}\right)\left(H_{l}, M_{\delta}\right) \\
& -\Phi(\alpha(q), u)(\zeta(\alpha(q)+u)-\zeta(u)) \sum_{\substack{\gamma, \delta \in \Delta \\
\gamma+\delta=\alpha}} N_{\gamma, \delta}\left(H_{k}, M_{\gamma}\right)\left(H_{l}, M_{\delta}\right) \\
& =\Phi(\alpha(q), u)(\zeta(\alpha(q)+u)-\zeta(\alpha(q))-\zeta(u)) \\
& \times\left(\left(H_{k}, H_{\alpha}\right)\left(H_{l}, M_{\alpha}^{+}\right)-\left(H_{k}, M_{\alpha}^{+}\right)\left(H_{l}, H_{\alpha}\right)\right. \\
& -2\left(2-\delta_{\theta \alpha,-\alpha}\right) \alpha\left(K_{\alpha}\right)\left(H_{k}, M_{\alpha}^{-}\right)\left(H_{l}, M_{\alpha}^{+}\right) \\
& \left.+2\left(2-\delta_{\theta \alpha,-\alpha}\right) \alpha\left(K_{\alpha}\right)\left(H_{k}, M_{\alpha}^{+}\right)\left(H_{l}, M_{\alpha}^{-}\right)\right) \\
& +\Phi(\alpha(q), u)(\zeta(\alpha(q)+u)-\zeta(u)) \\
& \times\left(+2\left(2-\delta_{\theta \alpha,-\alpha}\right) \alpha\left(K_{\alpha}\right)\left(H_{k}, M_{\alpha}^{-}\right)\left(H_{l}, M_{\alpha}^{+}\right)\right. \\
& -2\left(2-\delta_{\theta \alpha,-\alpha}\right) \alpha\left(K_{\alpha}\right)\left(H_{k}, M_{\alpha}^{+}\right)\left(H_{l}, M_{\alpha}^{-}\right) \\
& \left.-\sum_{\substack{\beta, \gamma \in \Delta \\
\beta+\gamma=\alpha}} N_{\beta, \gamma}\left(H_{k}, M_{\beta}\right)\left(H_{l}, M_{\gamma}\right)\right) \\
& \begin{aligned}
+\Phi(\alpha(q), u) \sum_{\substack{\beta \in \Delta \\
\beta \neq \pm \alpha, \beta \neq \pm \theta \alpha}} \zeta(\beta(q))\left(H_{k}, M_{\beta}\right) \\
\quad\left(H_{l}, \alpha\left(K_{-\beta}\right) M_{\alpha}+\beta\left(M_{\alpha}\right) M_{-\beta}+N_{\beta, \alpha-\beta} M_{\alpha-\beta}\right)
\end{aligned} \\
& -\Phi(\alpha(q), u) \sum_{\beta \in \Delta} \zeta(\beta(q))\left(H_{l}, M_{\beta}\right) \\
& { }^{\beta \neq \pm \alpha, \beta \neq \pm \theta \alpha} \times\left(H_{k}, \alpha\left(K_{-\beta}\right) M_{\alpha}+\beta\left(M_{\alpha}\right) M_{-\beta}-N_{\alpha-\beta, \beta} M_{\alpha-\beta}\right) \\
& -\left(1-\delta_{\theta \alpha,-\alpha}\right) \Phi(\alpha(q), u) \zeta(\alpha(q))\left(+N_{\theta \alpha, \alpha-\theta \alpha}\left(H_{k}, M_{\theta \alpha}\right)\left(H_{l}, M_{\alpha-\theta \alpha}\right)\right. \\
& -N_{-\theta \alpha, \alpha+\theta \alpha}\left(H_{k}, M_{-\theta \alpha}\right)\left(H_{l}, M_{\alpha+\theta \alpha}\right) \\
& +N_{\alpha-\theta \alpha, \theta \alpha}\left(H_{l}, M_{\theta \alpha}\right)\left(H_{k}, M_{\alpha-\theta \alpha}\right) \\
& \left.-N_{\alpha+\theta \alpha,-\theta \alpha}\left(H_{l}, M_{-\theta \alpha}\right)\left(H_{k}, M_{\alpha+\theta \alpha}\right)\right) \text {. }
\end{aligned}
$$

The last term vanishes due to the condition (148), whereas the previous two terms vanish due to eqn (142). The first term also vanishes because, as already observed before, $M_{\alpha}^{+}=0$ for real roots $\alpha$ while, according to eqns (133), (134) and (146),

$$
4 \alpha\left(K_{\alpha}\right) M_{\alpha}^{-}=4 \alpha\left(K_{\alpha}^{-}\right) M_{\alpha}^{-}=4 \alpha\left(M_{\alpha}^{-}\right) M_{\alpha}^{-}=\left(H_{\alpha}\right)_{\mathfrak{a}}
$$


for complex roots $\alpha$. Finally, the same reasoning shows that the second term will vanish provided we assume that

$$
\sum_{\substack{\beta, \gamma \in \Delta \\ \beta+\gamma=\alpha}} N_{\beta, \gamma} M_{\beta} \otimes M_{\gamma}=\frac{\epsilon_{\alpha}}{\sqrt{2}}|\alpha|\left(M_{\alpha} \otimes M_{-\alpha}-M_{-\alpha} \otimes M_{\alpha}\right),
$$

which is easily reduced to the second equation in eqn (125) by noting that $\frac{1}{2}\left(M_{\alpha} \otimes M_{-\alpha}-M_{-\alpha} \otimes M_{\alpha}\right)=M_{\alpha}^{-} \otimes M_{\alpha}^{+}-M_{\alpha}^{+} \otimes M_{\alpha}^{-}=M_{\alpha}^{-} \otimes M_{\alpha}-M_{\alpha} \otimes M_{\alpha}^{-}$ and using eqn (122). Note that in the degenerate case, the same argument works, but eqn (152) is not needed.

The proof of eqn (97) proceeds along similar lines, using the functional equations (29)-(31):

$$
\begin{aligned}
& \alpha_{k} \Phi^{\prime}(\alpha(q), v) K_{\alpha} \\
&+\frac{1}{2}(\zeta(u-v)+\zeta(u+v)) A_{k}^{\alpha}(q, v)\left(H_{\alpha}\right)_{\mathfrak{a}} \\
&+ \frac{1}{2}(\zeta(u-v)-\zeta(u+v)+2 \zeta(v)) A_{k}^{\alpha}(q, v)\left(H_{\alpha}\right)_{\mathfrak{b}} \\
&+ \Phi(\alpha(q), v) \alpha\left(A_{k}^{\mathfrak{h}}(q, v)\right) K_{\alpha}-\sum_{\substack{\gamma, \delta \in \Delta \\
\gamma+\delta=\alpha}} N_{\gamma, \delta} \Phi(\gamma(q), v) A_{k}^{\delta}(q, v) K_{\gamma} \\
&+ \frac{1}{2} \Phi(\alpha(q), v-u) A_{k}^{\alpha}(q, u) H_{\alpha}+\frac{1}{2} \Phi(\alpha(q), v+u) A_{k}^{\alpha}(q,-u) \theta H_{\alpha} \\
&+ \sum_{j=1}^{r} \alpha_{j} A_{k}^{\alpha}(q, v) A_{j}^{\mathfrak{h}}(q, u) \\
&= \Phi^{\prime}(\alpha(q), v) \alpha_{k} K_{\alpha} \\
&+\frac{1}{2} \Phi(\alpha(q), v)(\zeta(u-v)+\zeta(u+v))\left(H_{k}, M_{\alpha}\right)\left(H_{\alpha}\right)_{\mathfrak{a}} \\
&+\frac{1}{2} \Phi(\alpha(q), v)(\zeta(u-v)-\zeta(u+v)+2 \zeta(v))\left(H_{k}, M_{\alpha}\right)\left(H_{\alpha}\right)_{\mathfrak{b}} \\
&+\Phi(\alpha(q), v)\left(\sum_{\beta \in \Delta} \zeta(\beta(q)) \alpha\left(K_{-\beta}\right)\left(H_{k}, M_{\beta}\right) K_{\alpha}-\zeta(v) \alpha_{k} K_{\alpha}\right) \\
&-\sum_{\gamma, \delta \in \Delta} N_{\gamma, \delta} \Phi(\gamma(q), v) \Phi(\delta(q), v)\left(H_{k}, M_{\delta}\right) K_{\gamma} \\
&+\frac{1}{2} \Phi(\alpha(q), v-u) \Phi(\alpha(q), u)\left(H_{k}, M_{\alpha}\right)\left(\left(H_{\alpha}\right)_{\mathfrak{a}}+\left(H_{\alpha}\right)_{\mathfrak{b}}\right) \\
&-\frac{1}{2} \Phi(\alpha(q), v+u) \Phi(\alpha(q),-u)\left(H_{k}, M_{\alpha}\right)\left(\left(H_{\alpha}\right)_{\mathfrak{a}}-\left(H_{\alpha}\right)_{\mathfrak{b}}\right)
\end{aligned}
$$




$$
\begin{aligned}
& -\Phi(\alpha(q), v) \sum_{j=1}^{r} \sum_{\beta \in \Delta} \zeta(\beta(q)) \alpha_{j}\left(H_{j}, M_{-\beta}\right)\left(H_{k}, M_{\alpha}\right) K_{\beta} \\
& -\Phi(\alpha(q), v) \zeta(u) \sum_{j=1}^{r} \alpha_{j}\left(H_{k}, M_{\alpha}\right) H_{j} \\
& =\left(\Phi^{\prime}(\alpha(q), v)-\Phi(\alpha(q), v) \zeta(v)\right)\left(H_{k}, H_{\alpha}\right) K_{\alpha} \\
& +\frac{1}{2}((\zeta(u-v)+\zeta(u+v)-2 \zeta(u)) \Phi(\alpha(q), v) \\
& +\Phi(\alpha(q), v-u) \Phi(\alpha(q), u) \\
& -\Phi(\alpha(q), v+u) \Phi(\alpha(q),-u))\left(H_{k}, M_{\alpha}\right)\left(H_{\alpha}\right)_{\mathfrak{a}} \\
& +\frac{1}{2}((\zeta(u-v)-\zeta(u+v)+2 \zeta(v)) \Phi(\alpha(q), v) \\
& +\Phi(\alpha(q), v-u) \Phi(\alpha(q), u) \\
& +\Phi(\alpha(q), v+u) \Phi(\alpha(q),-u))\left(H_{k}, M_{\alpha}\right)\left(H_{\alpha}\right)_{\mathfrak{b}} \\
& +\Phi(\alpha(q), v) \zeta(\alpha(q))\left(+\alpha\left(K_{-\alpha}\right)\left(H_{k}, M_{\alpha}\right) K_{\alpha}-\alpha\left(K_{\alpha}\right)\left(H_{k}, M_{-\alpha}\right) K_{\alpha}\right. \\
& \text { - }\left(1-\delta_{\theta \alpha,-\alpha}\right) \alpha\left(K_{-\theta \alpha}\right)\left(H_{k}, M_{\theta \alpha}\right) K_{\alpha} \\
& +\left(1-\delta_{\theta \alpha,-\alpha}\right) \alpha\left(K_{\theta \alpha}\right)\left(H_{k}, M_{-\theta \alpha}\right) K_{\alpha} \\
& -\alpha\left(M_{-\alpha}\right)\left(H_{k}, M_{\alpha}\right) K_{\alpha}+\alpha\left(M_{\alpha}\right)\left(H_{k}, M_{\alpha}\right) K_{-\alpha} \\
& +\left(1-\delta_{\theta \alpha,-\alpha}\right) \alpha\left(M_{-\theta \alpha}\right)\left(H_{k}, M_{\alpha}\right) K_{\theta \alpha} \\
& \left.-\left(1-\delta_{\theta \alpha,-\alpha}\right) \alpha\left(M_{\theta \alpha}\right)\left(H_{k}, M_{\alpha}\right) K_{-\theta \alpha}\right) \\
& +\Phi(\alpha(q), v) \sum_{\substack{\beta \in \Delta \\
\beta \neq \pm \alpha, \beta \neq \pm \theta \alpha}} \zeta(\beta(q))\left(H_{k}, M_{\beta}\right)\left(\alpha\left(K_{-\beta}\right) K_{\alpha}+\underline{\beta\left(K_{\alpha}\right) K_{-\beta}}\right) \\
& -\Phi(\alpha(q), v) \sum_{\substack{\beta \in \Delta \\
\beta \neq \pm \alpha, \beta \neq \pm \theta \alpha}} \zeta(\beta(q))\left(H_{k}, \alpha\left(M_{-\beta}\right) M_{\alpha}+\underline{\beta\left(K_{\alpha}\right) M_{-\beta}}\right) K_{\beta} \\
& -\Phi(\alpha(q), v) \sum_{\substack{\gamma, \delta \in \Delta \\
\gamma+\delta=\alpha}}(\zeta(\gamma(q))+\zeta(\delta(q))) N_{\gamma, \delta}\left(H_{k}, M_{\delta}\right) K_{\gamma} \\
& +\Phi(\alpha(q), v)(\zeta(\alpha(q)+v)-\zeta(v)) \sum_{\substack{\gamma, \delta \in \Delta \\
\gamma+\delta=\alpha}} N_{\gamma, \delta}\left(H_{k}, M_{\delta}\right) K_{\gamma}
\end{aligned}
$$




$$
\begin{aligned}
& =\Phi(\alpha(q), v)(\zeta(\alpha(q)+v)-\zeta(\alpha(q))-\zeta(v)) \\
& \times\left(\left(H_{k}, H_{\alpha}\right) K_{\alpha}-\left(H_{k}, M_{\alpha}\right)\left(H_{\alpha}\right)_{\mathfrak{b}}\right. \\
& -\left(2-\delta_{\theta \alpha,-\alpha}\right)\left(\alpha\left(K_{-\alpha}\right)-\alpha\left(M_{-\alpha}\right)\right)\left(H_{k}, M_{\alpha}\right) K_{\alpha} \\
& +\left(2-\delta_{\theta \alpha,-\alpha}\right) \alpha\left(K_{\alpha}\right)\left(H_{k}, M_{-\alpha}\right) K_{\alpha} \\
& \left.-\left(2-\delta_{\theta \alpha,-\alpha}\right) \alpha\left(M_{\alpha}\right)\left(H_{k}, M_{\alpha}\right) K_{-\alpha}\right) \\
& +\Phi(\alpha(q), v)(\zeta(\alpha(q)+v)-\zeta(v)) \\
& \times\left(+\left(2-\delta_{\theta \alpha,-\alpha}\right)\left(\alpha\left(K_{-\alpha}\right)-\alpha\left(M_{-\alpha}\right)\right)\left(H_{k}, M_{\alpha}\right) K_{\alpha}\right. \\
& -\left(2-\delta_{\theta \alpha,-\alpha}\right) \alpha\left(K_{\alpha}\right)\left(H_{k}, M_{-\alpha}\right) K_{\alpha} \\
& +\left(2-\delta_{\theta \alpha,-\alpha}\right) \alpha\left(M_{\alpha}\right)\left(H_{k}, M_{\alpha}\right) K_{-\alpha} \\
& \left.-\sum_{\substack{\beta, \gamma \in \Delta \\
\beta+\gamma=\alpha}} N_{\beta, \gamma}\left(H_{k}, M_{\beta}\right) K_{\gamma}\right) \\
& \begin{aligned}
+\Phi(\alpha(q), v) & \sum_{\substack{\beta \in \Delta \\
\beta \neq \pm \alpha, \beta \neq \pm \theta \alpha}} \zeta(\beta(q)) \\
& \\
&
\end{aligned} \\
& \begin{aligned}
-\Phi(\alpha(q), v) & \sum_{\substack{\beta \in \Delta \\
\beta \neq \pm \alpha, \beta \neq \pm \theta \alpha}} \zeta(\beta(q)) \\
& \left(H_{k}, \alpha\left(M_{-\beta}\right) M_{\alpha}+\beta\left(K_{\alpha}\right) M_{-\beta}+N_{\beta, \alpha-\beta} M_{\alpha-\beta}\right) K_{\beta}
\end{aligned} \\
& +\left(1-\delta_{\theta \alpha,-\alpha}\right) \Phi(\alpha(q), v) \zeta(\alpha(q))\left(+N_{\alpha-\theta \alpha, \theta \alpha}\left(H_{k}, M_{\theta \alpha}\right) K_{\alpha-\theta \alpha}\right. \\
& -N_{\alpha+\theta \alpha,-\theta \alpha}\left(H_{k}, M_{-\theta \alpha}\right) K_{\alpha+\theta \alpha} \\
& +N_{\theta \alpha, \alpha-\theta \alpha}\left(H_{k}, M_{\alpha-\theta \alpha}\right) K_{\theta \alpha} \\
& \left.-N_{-\theta \alpha, \alpha+\theta \alpha}\left(H_{k}, M_{\alpha+\theta \alpha}\right) K_{-\theta \alpha}\right) \text {. }
\end{aligned}
$$

The last term vanishes due to the condition (148), whereas the previous two terms vanish due to eqn (142) and provided we impose the relation

$$
\begin{gathered}
\alpha\left(K_{\beta}\right) K_{\alpha}-\frac{1}{2}\left(N_{\alpha, \beta} K_{\alpha+\beta}+N_{\theta \alpha, \beta} K_{\theta \alpha+\beta}\right)-\beta\left(K_{\alpha}\right) K_{\beta}=0 \\
\text { for } \alpha, \beta \in \Delta \text { such that } \beta \neq \pm \alpha, \beta \neq \pm \theta \alpha
\end{gathered}
$$

which is complementary to it. The first term also vanishes because, according to equs (149) and (150), 


$$
\begin{aligned}
\left(H_{k},\right. & \left.H_{\alpha}\right) K_{\alpha}-\left(H_{k}, M_{\alpha}\right)\left(H_{\alpha}\right)_{\mathfrak{b}} \\
& =\left(H_{k}, H_{\alpha}\right) K_{\alpha}^{+}-\left(H_{k}, M_{\alpha}^{+}\right)\left(H_{\alpha}\right)_{\mathfrak{b}} \\
& =\epsilon_{\alpha} \sqrt{2}|\alpha|\left(\left(H_{k}, M_{\alpha}^{-}\right) K_{\alpha}^{+}-\left(H_{k}, M_{\alpha}^{+}\right) K_{\alpha}^{-}\right) \\
& =\frac{\epsilon_{\alpha}}{\sqrt{2}}|\alpha|\left(\left(H_{k}, M_{\alpha}\right) K_{-\alpha}-\left(H_{k}, M_{-\alpha}\right) K_{\alpha}\right),
\end{aligned}
$$

whereas for real roots $\alpha \in \Delta(\theta \alpha=-\alpha)$, we have $K_{-\alpha}=K_{\alpha}, M_{-\alpha}=-M_{\alpha}, \alpha\left(K_{ \pm \alpha}\right)=0$ and hence by eqn (145)

$$
\begin{aligned}
- & \left(2-\delta_{\theta \alpha,-\alpha}\right)\left(\alpha\left(K_{-\alpha}\right)-\alpha\left(M_{-\alpha}\right)\right)\left(H_{k}, M_{\alpha}\right) K_{\alpha} \\
+ & \left(2-\delta_{\theta \alpha,-\alpha}\right) \alpha\left(K_{\alpha}\right)\left(H_{k}, M_{-\alpha}\right) K_{\alpha} \\
- & \left(2-\delta_{\theta \alpha,-\alpha}\right) \alpha\left(M_{\alpha}\right)\left(H_{k}, M_{\alpha}\right) K_{-\alpha} \\
= & \alpha\left(M_{-\alpha}\right)\left(H_{k}, M_{\alpha}\right) K_{\alpha}-\alpha\left(M_{\alpha}\right)\left(H_{k}, M_{\alpha}\right) K_{-\alpha} \\
& =-\frac{\epsilon_{\alpha}}{\sqrt{2}}|\alpha|\left(\left(H_{k}, M_{\alpha}\right) K_{-\alpha}-\left(H_{k}, M_{-\alpha}\right) K_{\alpha}\right),
\end{aligned}
$$

while for complex roots $\alpha \in \Delta(\theta \alpha \neq \pm \alpha)$, we have $\alpha\left(K_{ \pm \alpha}\right)=\alpha\left(M_{ \pm \alpha}\right)$ and hence by eqn (146)

$$
\begin{aligned}
- & \left(2-\delta_{\theta \alpha,-\alpha}\right)\left(\alpha\left(K_{-\alpha}\right)-\alpha\left(M_{-\alpha}\right)\right)\left(H_{k}, M_{\alpha}\right) K_{\alpha} \\
+ & \left(2-\delta_{\theta \alpha,-\alpha}\right) \alpha\left(K_{\alpha}\right)\left(H_{k}, M_{-\alpha}\right) K_{\alpha} \\
- & \left(2-\delta_{\theta \alpha,-\alpha}\right) \alpha\left(M_{\alpha}\right)\left(H_{k}, M_{\alpha}\right) K_{-\alpha} \\
& =2 \alpha\left(M_{\alpha}\right)\left(H_{k}, M_{-\alpha}\right) K_{\alpha}-2 \alpha\left(M_{\alpha}\right)\left(H_{k}, M_{\alpha}\right) K_{-\alpha} \\
& =-\frac{\epsilon_{\alpha}}{\sqrt{2}}|\alpha|\left(\left(H_{k}, M_{\alpha}\right) K_{-\alpha}-\left(H_{k}, M_{-\alpha}\right) K_{\alpha}\right) .
\end{aligned}
$$

Finally, the same reasoning shows that the second term will vanish provided we assume that

$$
\sum_{\substack{\beta, \gamma \in \Delta \\ \beta+\gamma=\alpha}} N_{\beta, \gamma} M_{\beta} \otimes K_{\gamma}=\frac{\epsilon_{\alpha}}{\sqrt{2}}|\alpha|\left(M_{\alpha} \otimes K_{-\alpha}-M_{-\alpha} \otimes K_{\alpha}\right)
$$

which is complementary to the condition (152) derived previously and is easily reduced to the first equation in eqn (125) by noting that

$$
\frac{1}{2}\left(M_{\alpha} \otimes K_{-\alpha}-M_{-\alpha} \otimes K_{\alpha}\right)=M_{\alpha}^{-} \otimes K_{\alpha}^{+}-M_{\alpha}^{+} \otimes K_{\alpha}^{-}=M_{\alpha}^{-} \otimes K_{\alpha}-M_{\alpha} \otimes K_{\alpha}^{-}
$$

and using eqn (122). Note that in the degenerate case, the same argument works, but eqn (154) is not needed. 
Having concluded the proof of Proposition 2, we pass to analyzing the implications of the algebraic constraints that we have derived. The first thing that suggests itself is to combine the generators $K_{\alpha}$ and $M_{\alpha}$ into generators

$$
F_{\alpha}=K_{\alpha}+M_{\alpha}
$$

which, according to eqns $(\underline{119})$ and $(\underline{120})$, define a $\theta$-covariant map from $\Delta$ to $\mathfrak{h}_{\mathbb{R}}$ :

$$
\theta F_{\alpha}=F_{\theta \alpha}
$$

Then eqn (121) becomes equivalent to eqn (51) and eqn (122) becomes equivalent to eqn (52). The relation between eqn (123) and eqn (53), however, is more intricate. To approach this question, note that eqn (53) decomposes naturally into a component along $\mathrm{ib}_{0}$,

$$
\begin{gathered}
\alpha\left(F_{\beta}\right) K_{\alpha}-\beta\left(F_{\alpha}\right) K_{\beta}=N_{\alpha, \beta} K_{\alpha+\beta} \\
\text { for } \alpha, \beta \in \Delta \text { such that } \beta \neq \pm \alpha, \beta \neq \pm \theta \alpha,
\end{gathered}
$$

and a component along $\mathfrak{a}_{0}$,

$$
\begin{gathered}
\alpha\left(F_{\beta}\right) M_{\alpha}-\beta\left(F_{\alpha}\right) M_{\beta}=N_{\alpha, \beta} M_{\alpha+\beta} \\
\text { for } \alpha, \beta \in \Delta \text { such that } \beta \neq \pm \alpha, \beta \neq \pm \theta \alpha .
\end{gathered}
$$

Now observe that eqn $(\overline{119})$ can be used to show that the second equation in eqn (123) is equivalent to eqn (158). Indeed, antisymmetrizing eqn (123) with respect to the exchange of $\alpha$ and $\beta$ eliminates one of the two terms containing structure constants and leads to eqn (158), and conversely, substituting $\alpha$ by $\theta \alpha$ in eqn (158) and subtracting the result, we are led back to the second equation in eqn (123). On the other hand, using eqn (120) and applying the same argument, the first equation in eqn (123) turns out to be a consequence of eqn (157) but is apparently weaker. Similarly, eqn (124) is part of eqn (54), from which it can be obtained by projecting from $\mathfrak{h}_{\mathbb{R}}$ onto $\mathfrak{a}_{0}$ in the third tensor factor, that is, by applying the operator $1 \otimes 1 \otimes \frac{1}{2}(1-\theta)$, and eqn (125) is part of eqn (55), from which it can be obtained by projecting from $\mathfrak{h}_{\mathbb{R}}$ onto $\mathfrak{a}_{0}$ in the first tensor factor, that is, by applying the operator $\frac{1}{2}(1-\theta) \otimes 1$.

Although the conditions stated in Proposition 2 thus seem to be weaker than those stated in Proposition 1, it turns out that they are still sufficiently strong to allow for a complete classification of all possible solutions. As a by-product, we shall be able to reduce eqn (122) to the form given in eqn (130). The arguments employed to achieve this are essentially the same as the ones in the previous section. First, we argue that, as before, the signs $\epsilon_{\alpha}$ that appear in eqn (122) may without loss of generality be assumed to be independent of $\alpha$. Next, writing down the system obtained from eqn (158) upon replacing $\alpha$ by $-\alpha$ and $\beta$ by $-\beta$, adding the resulting four equations, inserting eqn (122) and separating the coefficients of $\left(H_{\alpha}\right)_{\mathfrak{a}}$ and $\left(H_{\beta}\right)_{\mathfrak{a}}$, we arrive at the same formula as in the previous section, eqn (78). Once again, it is to be noted that this derivation is only valid when $\beta \neq \pm \alpha, \beta \neq \pm \theta \alpha$, as stated in eqn (158): this supplementary condition is 
also needed to guarantee that $\left(H_{\alpha}\right)_{\mathfrak{a}}$ and $\left(H_{\beta}\right)_{\mathfrak{a}}$ are linearly independent but can in fact be eliminated from eqn (78) since this formula is automatically satisfied when $\beta= \pm \alpha$ or $\beta= \pm \theta \alpha$. (Indeed, for $\beta= \pm \alpha$ or $\beta= \pm \theta \alpha$ the rhs is understood to vanish since $2 \alpha, 0$ and $\alpha \pm \theta \alpha$ do not belong to the root system $\Delta$, whereas the lhs vanishes as a consequence of eqn (121).)

The statement that for $\beta \neq \pm \alpha$ and $\beta \neq \pm \theta \alpha$, the generators $\left(H_{\alpha}\right)_{\mathfrak{a}}$ and $\left(H_{\beta}\right)_{\mathfrak{a}}$ are linearly independent, used in the derivation of eqn $(78)$ given here, can be proved indirectly, as follows. Suppose that for some pair of roots $\alpha, \beta \in \Delta$ satisfying $\beta \neq \pm \alpha$ and $\beta \neq \pm \theta \alpha$, these generators were linearly dependent. Since $\Delta_{0}$ is empty so that $\left(H_{\alpha}\right)_{\mathfrak{a}}$ and $\left(H_{\beta}\right)_{\mathfrak{a}}$ are both non-zero, this amounts to assuming that there exists a non-zero real number $\lambda$ such that $\left(H_{\beta}\right)_{\mathfrak{a}}=\lambda\left(H_{\alpha}\right)_{\mathfrak{a}}$, or equivalently,

$$
\beta-\theta \beta=\lambda(\alpha-\theta \alpha) .
$$

Obviously, if both roots are real, eqn (159) reduces to $\beta=\lambda \alpha$, with $\lambda= \pm 1$, a contradiction. Similarly, if one of the two roots is complex while the other is real, we also get a contradiction since if, for example, $\alpha$ is complex and $\beta$ is real, eqn (159) becomes $\beta=\frac{1}{2} \lambda(\alpha-\theta \alpha)$ which is excluded since $\alpha$ and $\theta \alpha$ being strongly orthogonal implies that the only linear combinations of $\alpha$ and $\theta \alpha$ which are roots are $\pm \alpha$ and $\pm \theta \alpha$. To handle the case where both roots are complex and hence $\alpha$ and $\theta \alpha$ as well as $\beta$ and $\theta \beta$ are strongly orthogonal, we begin by noting that $\beta$ cannot be orthogonal to both $\alpha$ and $\theta \alpha$ since otherwise, $\theta \beta$ would be so as well and hence $\beta-\theta \beta$ would be orthogonal to $\alpha-\theta \alpha$, which contradicts eqn (159). Exchanging $\alpha$ with $\theta \alpha$ and $\beta$ with $\theta \beta$ if necessary, we may assume without loss of generality that $\alpha$ is not orthogonal to $\beta$ and that the factor $\lambda$ in eqn (159) is positive. With these conventions, taking the scalar product of eqn (159) with $\alpha$ and with $\beta$ gives

$$
\begin{gathered}
(\beta, \alpha)-(\theta \beta, \alpha)=\lambda(\alpha, \alpha), \\
(\beta, \beta)=\lambda(\beta, \alpha)-\lambda(\beta, \theta \alpha),
\end{gathered}
$$

implying

$$
(\beta, \beta)=\lambda^{2}(\alpha, \alpha) .
$$

In the root system of an arbitrary simple complex Lie algebra, this forces $\lambda^{2}$ to be $1,2,3, \frac{1}{2}$ or $\frac{1}{3}$. But eqn (159) excludes the possibility of $\lambda^{2}$ being different from 1 since the root system of any simple complex Lie algebra is contained in an appropriate lattice formed by the integer linear combinations of vectors $\frac{1}{2} e_{i}$ where the $e_{i}$ are an orthonormal basis of $\mathbb{R}^{n}$, so an equation of the form (159) with an irrational value of $\lambda$ can only hold if both sides vanish, which is impossible since $\Delta_{0}$ is empty. Thus we conclude that $\lambda=1$, so $\alpha, \beta, \theta \alpha$ and $\theta \beta$ all have the same length and eqn (159) becomes

$$
\beta-\theta \beta=\alpha-\theta \alpha
$$


This allows us to determine the $\alpha$-string through $\beta$. First, $\beta-\alpha$ cannot be a root since if it were, it would belong to $\Delta_{0}$ which is empty. Second, $\beta+\alpha$ must therefore be a root, since $\alpha$ and $\beta$ are not orthogonal. Third, $\beta+2 \alpha$ cannot be a root since if it were, we would have $2(\beta, \alpha) /(\alpha, \alpha) \leqslant-2$, implying $|\beta+\alpha|^{2} \leqslant 0$, which is absurd. Hence $\alpha$ and $\beta$ generate a root system of type $A_{2}$ for which they act as simple roots; in particular, $2(\beta, \alpha) /(\alpha, \alpha)=-1$. Inserting this conclusion back into eqn (160), we see that $2(\theta \beta, \alpha) /(\alpha, \alpha)=-3$, which is only possible if the $\alpha$-string through $\theta \beta$ consists of four roots, namely $\theta \beta, \theta \beta+\alpha, \theta \beta+2 \alpha$ and $\theta \beta+3 \alpha$ (recall that any root string has length at most 4 ). But this requires the angle between $\theta \beta$ and $\alpha$ to be $-150^{\circ}$ and forces $\theta \beta$ and $\alpha$ to have different length, contrary to a conclusion reached before.

In this way, we arrive once again at the conclusion that the simple complex Lie algebra $\mathfrak{g}$ must belong to the $A$-series. Moreover, the automorphism $\theta$ that defines the symmetric pair $(\mathfrak{g}, \theta)$ is further restricted by various additional constraints. The first such condition is that the root generators $E_{\alpha}$ in $\mathfrak{g}$ can be chosen so that $\theta E_{\alpha}=E_{\theta \alpha}$ for all $\alpha \in \Delta$, which according to the erratum of Ref. [1] is not only sufficient but also necessary to guarantee that the proof of integrability given in Ref. [1] really works: this excludes the symmetric pairs of the $A I$-series $S L(n, \mathbb{R}) / S O(n)$ for which all roots are real and $\theta E_{\alpha}=-E_{\theta \alpha}$ for all $\alpha \in \Delta$. The second condition is that there should be no imaginary roots: this excludes the symmetric pairs of the $A I I$-series $S L(n, \mathbb{H}) / S p(n)$ as well as the symmetric pairs of the $A I I I$ - series of complex Grassmannians $S U(p, q) / S(U(p) \times U(q))$ with $|p-q| \geqslant 1$. The third and final condition is that for all complex roots $\alpha, \theta \alpha$ should be strongly orthogonal to $\alpha$ : this excludes the symmetric pairs of the $A I I I$ series of complex Grassmannians $S U(p, q) / S(U(p) \times U(q))$ with $p \neq q$. On the other hand, it is clear that the symmetric pairs associated with the Grassmannians $S U(n, n) / S(U(n) \times U(n))$ do provide a non-trivial solution: explicitly, we have in the notation employed at the end of the previous section (with $n$ replaced by $2 n$ ) and in Sect. 3.2 of Ref. [四]

$$
\begin{gathered}
K_{a b}^{+}=-\frac{1}{4}\left(E_{a a}+E_{b b}+E_{\theta(a) \theta(a)}+E_{\theta(b) \theta(b)}\right)+\frac{1}{2 n} \mathbf{1}_{2 n}, \\
M_{a b}^{+}=-\frac{1}{4}\left(E_{a a}+E_{b b}-E_{\theta(a) \theta(a)}-E_{\theta(b) \theta(b)}\right)
\end{gathered}
$$

and

$$
\begin{aligned}
& K_{a b}^{-}=\frac{\epsilon}{4}\left(E_{a a}-E_{b b}+E_{\theta(a) \theta(a)}-E_{\theta(b) \theta(b)}\right), \\
& M_{a b}^{-}=\frac{\epsilon}{4}\left(E_{a a}-E_{b b}-E_{\theta(a) \theta(a)}+E_{\theta(b) \theta(b)}\right),
\end{aligned}
$$


implying that

$$
K_{a b}=-\frac{1}{2}\left(E_{b b}+E_{\theta(b) \theta(b)}\right)+\frac{1}{2 n} \mathbf{1}_{2 n}, \quad M_{a b}=-\frac{1}{2}\left(E_{b b}-E_{\theta(b) \theta(b)}\right)
$$

when $\epsilon=+1$, while

$$
K_{a b}=-\frac{1}{2}\left(E_{a a}+E_{\theta(a) \theta(a)}\right)+\frac{1}{2 n} \mathbf{1}_{2 n}, M_{a b}=-\frac{1}{2}\left(E_{a a}-E_{\theta(a) \theta(a)}\right)
$$

when $\epsilon=-1$.

\section{Conclusions and Outlook}

Our analysis of the question whether the known dynamical $R$-matrices for integrable Calogero models can be gauge transformed to numerical $R$-matrices has revealed that this is possible in some cases but not in all - a conclusion that could definitely not be reached by looking at the standard model associated with the root system of the $A$-series alone. In fact, it had been known from previous work that a) the Calogero models associated with the root systems of simple complex Lie algebras $\mathfrak{g}$ are integrable, in the sense of admitting a Lax representation with a dynamical $R$-matrix, if and only if $\mathfrak{g}=\mathfrak{s l}(n, \mathbb{C})$ [1] and b) that this dynamical $R$-matrix can be gauge transformed to a numerical one [5, 6]. The results reported in this paper show that for the Calogero models associated with the root systems of symmetric pairs $(\mathfrak{g}, \theta)$, the situation is more intricate. First of all, there is still no complete answer to the question which of these models are integrable, in the sense of admitting a Lax representation with a dynamical $R$-matrix: the only case that has been analyzed completely is that of the $A I I I$-series of complex Grassmannians $S U(p, q) / S(U(p) \times U(q))$, where integrability has been shown to occur if and only if $|p-q|$ is either 0 or 1 . We strongly suspect that this is in fact the only class of symmetric spaces where integrability prevails, but a rigorous proof of this conjecture is still missing. What is shown in this paper is that dynamical $R$-matrices of integrable Calogero models associated with non-Grassmannian symmetric pairs should they exist - cannot be gauge transformed to numerical $R$-matrices and, more importantly, that the dynamical $R$-matrices of the Grassmannian Calogero models can be gauge transformed to numerical $R$-matrices if $p=q$ but not if $|p-q|=1$. The first case includes the $C_{n}$ and $D_{n}$ models, whereas the second case includes the $B_{n}$ and $B C_{n}$ models.

In summary, our results show that the question which originally motivated our work on integrability of the Calogero models, namely the search for an understanding of the mathematical nature and role of dynamical $R$-matrices, is still far from a definite answer, since the attempt to reduce them to numerical $R$-matrices via gauge transformations is only partially successful. 
Accepting the fact that the role of dynamical $R$-matrices for our understanding of integrable systems can apparently not be reduced to that of numerical $R$-matrices in disguise, there are many questions that gain new impetus. Continuing to use the Calogero models as a guideline, we believe that there are several directions in which future work will be capable of providing new insights into the problem. One of them is the question of what should be the algebro-differential constraints to be satisfied by a truly dynamical $R$-matrix, or in other words, what is the real mathematical status and interpretation of the dynamical Yang-Baxter equation. A remarkable fact is that, as will be shown in a separate publication [10], there is a natural candidate which is

gauge invariant. Another promising direction for research is a further clarification of the relation between Calogero models and the geodesic flow on symmetric spaces subjected to Marsden-Weinstein phase space reduction: this relation should also shed new light on the role of the recently introduced spin Calogero models [11].

\section{References}

[1] M. Forger and A. Winterhalder: Dynamical R-Matrices for Calogero Models, Nucl. Phys. B 621 [PM] (2002) 523-570.

[2] M.A. Olshanetsky and A.M. Perelomov: Completely Integrable Hamiltonian Systems Connected with Semisimple Lie Algebras, Invent. Math. 37 (1976) 93-108.

[3] M.A. Olshanetsky and A.M. Perelomov: Classical Integrable Finite-Dimensional Systems Related to Lie Algebras, Phys. Rep. 71 (1981) 313-400.

[4] A.M. Perelomov: Integrable Systems of Classical Mechanics and Lie Algebras, Vol. 1 (Birkhäuser, Basel, 1990).

[5] B.Y. Hou and W.L. Yang: The Non-Dynamical R-Matrix Structure of the Elliptic Calogero-Moser Model, Lett. Math. Phys. 44 (1998) 35-41, solv-int/9711008, The Non-Dynamical R-Matrix Structure of the Elliptic $A_{n-1}$ Calogero-Moser Model, J. Phys. A - Math. Gen. 32 (1999) 1475-1486, q-alg/9711010.

[6] L. Fehér and B.G. Pusztai: On the Classical R-Matrix of the Degenerate CalogeroMoser Models, Czech J. Phys. 50 (2000) 59-64, math-ph/9912021v2, The NonDynamical R-Matrices of the Degenerate Calogero-Moser Models, J. Phys. A Math. Gen. 33 (2000) 7739-7759, math-ph/0005021v2.

[7] O. Babelon and C.-M. Viallet: Hamiltonian Structures and Lax Equations, Phys. Lett. B 237 (1990) 411-416.

[8] A.W. Knapp: Lie Groups Beyond an Introduction, Birkhäuser Verlag, Basel 1996.

[9] S. Helgason: Differential Geometry, Lie Groups and Symmetric Spaces, Academic Press, New York 1978. 
[10] M. Forger and A. Winterhalder: Gauge Transformations of the Dynamical YangBaxter Equation, in preparation.

[11] Luen-Chau Li and Ping Xu: Spin Calogero-Moser Systems Associated with Simple Lie Algebras, C. R. Acad. Sci. Paris Série I 331 (2000) 55-60, math.SG/0009180, Integrable Spin Calogero-Moser Systems, math.QA/0105162. 